\title{
The patterning behaviour of gravitationally modulated supercritical Marangoni flow in liquid layers
}

\author{
Marcello Lappa \\ Department of Mechanical and Aerospace Engineering, University of Strathclyde \\ James Weir Building, 75 Montrose Street, Glasgow, G1 1XJ, UK \\ email: marcello.lappa@strath.ac.uk-marlappa@unina.it
}

\begin{abstract}
The objective of the present analysis is the investigation of "hybrid convection" induced by the joint influence of imposed vibrations (g-jitters) of desired amplitude and frequency and surface-tension-induced forces in a non-isothermal liquid layer. This study may be regarded as the natural extension of an earlier work by other authors (Shevtsova, Nepomnyashchy and Legros, Phys. Rev. E 67, 066308, 2003) where the focus was on convection driven by interacting thermocapillarity and steady gravity. As in that work, conditions are considered for which the unperturbed (vibration-less) Marangoni flow would be characterized by the emergence and propagation of a classical hydrothermal wave, namely, a supercritical thermofluidynamic disturbance propagating continuously in the upstream direction. A number of numerical results are analysed and discussed. Regimes of quasi-stationary rolls, standing waves, traveling waves and modulated (pulso-traveling) disturbances are identified in the considered space of parameters. Most interestingly, it is observed that traveling waves can reverse their direction of propagation in some specific regions of the phase space.
\end{abstract}

PACS: 47.20.Dr, 47.20.Ky, 47.54.-r,47.11.Bc

\section{Introduction}

In the present work we consider "mixed convection" induced by the joint influence of surfacetension-induced forces and imposed vibrations (g-jitters) of desired amplitude and frequency in a non-isothermal liquid layer.

The latter kind of convection, generally referred to as "thermovibrational flow", initially studied due to its perturbing and undesired influence on microgravity experiments [1,2], has recently witnessed a renewed and significant theoretical interest because it has been understood that adequate knowledge of it may be used for the elaboration of a new strategy of flow control.

The use of vibrations may be regarded as a rather new and yet less investigated technique to be used more universally (Simonenko and Zen'kovskaja [3]) than traditional methods used in crystal growth and other technological processes because it is not restricted to electrically conductive melts (as is the case for magnetic fields). Vibrations and related effects could be applied without limitations for effective flow control also in situations in which the working fluid is organic.

A proper introduction of the topic considered in the present work and its collocation in a relevant research historical background should perhaps start from the simple remark that although many studies have been appearing in the literature over the last two or three decades for "pure" Marangoni flow (both theoretical and experimental, see, e.g., Zebib et al. [4], Bucchignani [5], Sato et al. [6] and, in particular, Schwabe [7] for rich and exhaustive reviews on the subject) and "pure" 
thermovibrational flow (Lyubimova et al. [8], Gabdrakhmanov and Kozlov [9], Savino and Lappa [10], Mialdun et al. [11], Shevtsova et al. [12]) the interaction dynamics of this these modes of convection are still obscure.

Some important analyses are available for the companion case of convection driven by interacting thermocapillarity and steady gravity, see e.g., Parmentier et al. [13], Burguete et al. [14] and the works by Nepomnyashchy and coworkers (Nepomnyashchy and Simanovskii [15,16], Shevtsova et al. [17], Nepomnyashchy et al. [18]). Despite the potential important applications mentioned above, however, studies expressly addressing the mixed thermocapillary-thermovibrational problem are rare and sparse ([19-30]). Moreover, most of existing numerical investigations were based on analytic solutions (valid under the assumption of an infinitely extended layer) and/or on the typical concepts and methods of the linear stability analysis.

Most recently, [31] reconsidered this specific subject addressing its potential application to the control of convection patterning and strength in shallow rectangular cavities of finite extent $(\mathrm{A}=$ length/height=4) with relevance to the most widely used technologies for the growth of singlecrystalline materials from the melt. Although the Navier-Stokes equations were solved in their complete and non-linear form, however, the numerical simulations were restricted to the case of low Prandtl number liquids (silicon, Pr=0.01), steady Marangoni flow and (an even more limiting assumption) vibrations of low amplitude and high frequency satisfying the conditions for which the socalled simplified Gershuni's mathematical model becomes applicable (by which the effective time required for the numerical simulations can be significantly shortened, Gershuni and Zhukhovitskii [32,33]). On the numerical side, some other developments deserve attention; among them the study by Grassia and Homsy [21,22], who considered the infinite parallel Marangoni flow subjected to gravitational modulation at low frequencies where the Gershuni's model is no longer applicable in various directions; these authors assumed as base unmodulated Marangoni flow the popular "return flow solution" and employed a quasi-steady approach, in the limit of a very low forcing frequency.

The new idea here is to somehow control the basic features of surface-tension-driven convection (not only the typical patterning behaviour in steady conditions but also the onset and properties of emerging oscillatory flow, etc.) in a geometry of finite extent via imposed vibrations without resorting to any specific assumptions on their frequency and amplitude.

From the theoretical viewpoint, it is important to mention that the dynamics of mixed thermocapillary-thermovibrational represent yet a rather complex challenge to researchers in the domain of fluid mechanics and heat transfer, because of the fact that it appears very difficult, if not to say practically impossible, to formulate any theory that can reasonably predict "a priori" the nonlinear properties of the resulting flow in terms of patterning behaviour and related waveforms. Numerical simulations are adversely affected in terms of computational resources and costs by the very high (both spatial and temporal) resolution required to capture the resulting delicate dynamics. A thorough understanding of the underlying physics is also still lacking because of their inherent complexities and involvement of different spatial and temporal scales. 


\section{Mathematical Model}

\section{A. The Geometry}

We consider a liquid layer with free liquid/gas interface parallel to the $\mathrm{x}$ axis and characteristic depth $\mathrm{L}=\mathrm{d}$ (Fig.1), laterally delimited by solid walls, one cooled, the other heated, adiabatic conditions on the remaining boundaries. The system aspect ratio (A), defined as its length-to-depth ratio ( $\ell / d$ ) is fixed to 20. The value of the Prandtl number ( $\operatorname{Pr}=v / \alpha$ where $v$ is the fluid kinematic viscosity and $\alpha$ is the thermal diffusivity) is $\operatorname{Pr}=15$. Moreover, no steady residual gravity is present. The vibrations are contained in the xy plane, being directed along a generic direction $\underline{\hat{n}}$ forming an angle $\theta$ with the free interface.

\section{B. The vibrations or g-jitters}

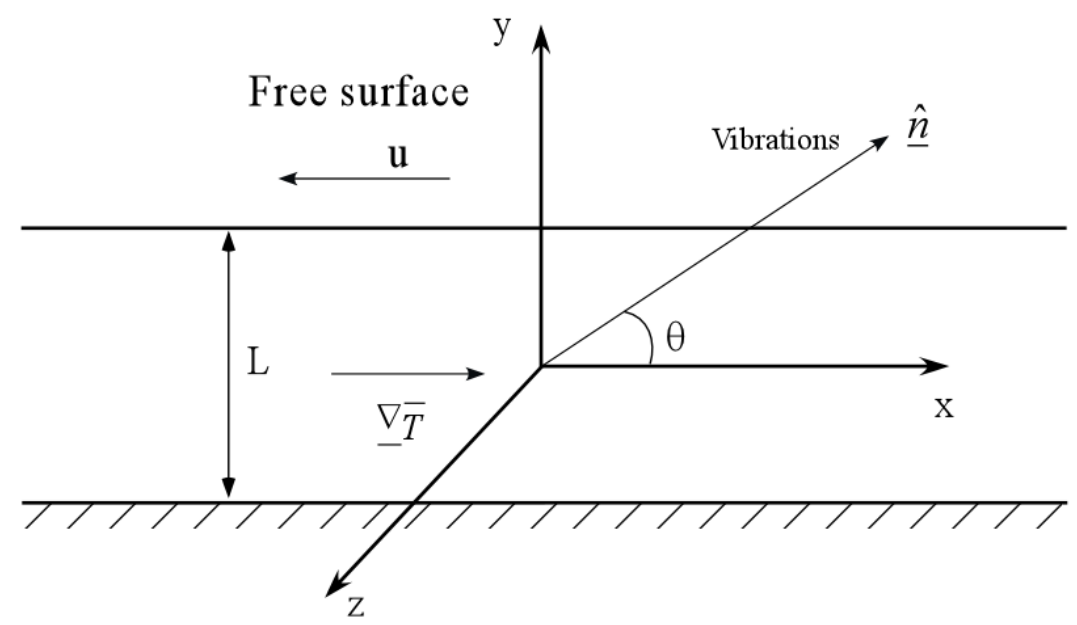

Figure 1: Sketch of fluid layer subjected to vibrations with arbitrary direction applied in the plane of the basic $2 \mathrm{D}$ flow.

By modelling vibrations as a sinusoidal displacement varying in time as $\underline{s}(t)=\operatorname{bsin}(\omega \mathrm{t}) \underline{\hat{n}}$, where $\mathrm{b}$ and $\omega(=2 \pi \mathrm{f})$ are its amplitude and angular frequency, respectively, it is known that, in the absence of other forces or effects and for a given fluid, i.e. for a fixed value of the Prandtl number, the properties of the emerging thermovibrational flow can be characterized in terms of two independent nondimensional parameters only, the nondimensional frequency ( $\varpi$ ) and the associated Rayleigh number $\left(\mathrm{Ra}_{\omega}\right)$ :

$\varpi=\frac{\omega L^{2}}{\alpha}$

$R a_{\omega}=\frac{b \omega^{2} \beta_{T} \Delta T L^{3}}{v \alpha}$

where $\beta_{\mathrm{T}}$ is the thermal expansion coefficient and $\Delta \mathrm{T}$ the imposed temperature difference. 
Since the displacement $\underline{s}(t)=\operatorname{bsin}(\omega \mathrm{t}) \underline{\hat{n}}$, can be considered exactly equivalent to an acceleration $\underline{g}(t)=\underline{g}_{\omega} \sin \left(\omega \mathrm{t}\right.$ ) (where $\underline{g}_{\omega}=\mathrm{b} \omega^{2} \underline{\hat{n}}$ ) and because the specificity of this alternating acceleration is that its linear effects have a zero-time averaged value, in the following we will use the terms "vibrations”, “system shaking or forcing”, “gravity modulation”, “periodic acceleration”, and “gjitters” as synonyms.

\section{The balance equations}

Scaling time, velocity, pressure and temperature by $\mathrm{L}^{2} / \alpha, \alpha / \mathrm{L}, \rho \alpha^{2} / \mathrm{L}^{2}$ (where $\rho$ is the fluid density) and $\Delta \mathrm{T}$, respectively, defining the temperature as $\mathrm{T}=\left(\overline{\mathrm{T}}-\overline{\mathrm{T}}_{\mathrm{m}}\right) / \Delta \mathrm{T}$ where $\bar{T}$ is the dimensional temperature and $\bar{T}_{m}$ a reference value, and using the canonical Boussinesq approximation to account for the effects of buoyancy, the nondimensional momentum equation can be written as:

$$
\frac{\partial \underline{V}}{\partial}=-\underline{\nabla} p-\underline{\nabla} \cdot[\underline{V} \underline{V}]+\operatorname{Pr} \nabla^{2} \underline{V}+\operatorname{Pr} R a_{\omega} T \sin (\varpi t) \underline{\hat{n}}
$$

Accordingly, the continuity and energy equations can be cast in compact form as:

$\underline{\nabla} \cdot \underline{\mathrm{V}}=0$

$$
\frac{\partial T}{\partial t}=-\underline{\nabla} \cdot[\underline{V T}]+\nabla^{2} T
$$

\section{The Boundary conditions}

Obviously, such equations must be supplemented with the adequate kinematic and thermal boundary conditions, which, by indicating with $\mathrm{u}$ and $\mathrm{v}$ the velocity components along $\mathrm{x}$ and $\mathrm{y}$, respectively, reduce to

$$
\begin{aligned}
& \mathrm{u}=0, \mathrm{v}=0 \text { and } \mathrm{T}= \pm 1 / 2 \text { at } \mathrm{x}= \pm \mathrm{A} / 2 \\
& \mathrm{u}=0, \mathrm{v}=0 \text { and } \partial \mathrm{T} / \partial \mathrm{y}=0 \text { at } \mathrm{y}=-1 / 2 \\
& \mathrm{v}=0, \partial \mathrm{u} / \partial \mathrm{y}=-\mathrm{Ma} \partial \mathrm{T} / \partial \mathrm{x} \text { and } \partial \mathrm{T} / \partial \mathrm{y}=0 \text { at } \mathrm{y}=1 / 2
\end{aligned}
$$

where $\mathrm{Ma}=\sigma_{\mathrm{T}} \Delta \mathrm{TL} / v \rho \alpha$

is the well-known Marangoni number ( $\sigma_{\mathrm{T}}$ being the derivative of the surface tension $\sigma$ with respect to temperature). Equation (5c) enforces a flow by tangential variation of the surface tension. The motion (thermocapillary or Marangoni convection) immediately results whenever a temperature gradient exists along the considered interface, no matter how small (see, e.g., [34,35]). 


\section{E. The Numerical method}

Balance equations and related boundary conditions (1-6) have been solved numerically by a timeexplicit finite-difference method (primitive-variable approach) based on a rectangular mesh and a staggered collocation of fluid-dynamic variables. In particular, forward differences in time and central-differencing schemes in space (second order accurate) have been used to discretize the energy and momentum governing equations. The related solution strategy is not discussed here, the interested reader being referred to various books and articles in the literature for an exhaustive treatment.

Here we limit ourselves just to providing some useful information about the grid refinement study and the strategy that we had to implement to guarantee a proper resolution of all spatial and temporal scales involved in the considered phenomena.

\begin{tabular}{rl}
\hline \hline Grid $\mathrm{N}_{\mathrm{x}} \times \mathrm{N}_{\mathrm{y}}$ & $\Omega_{\mathrm{HTW}}$ \\
\hline \hline $200 \times 20$ & 50.4 \\
$400 \times 20$ & 48.0 \\
$300 \times 30$ & 47.5 \\
$400 \times 30$ & 47.2 \\
$600 \times 30$ & 47.1 \\
\hline \hline
\end{tabular}

Table I: Grid Refinement Study: Angular frequency of the Hydrothermal Wave as a function of mesh resolution $\left(\mathrm{Pr} \cong 15, \mathrm{~A}=20, \mathrm{Ma}=3 \times 10^{4}\right)$

As sensitive parameters for the spatial grid refinement study, we have considered the angular frequency of the hydrothermal wave representing the typical supercritical state of pure Marangoni flow (see Sect. IIIA). The minimum mesh required to guarantee independency of such a frequency from the used spatial resolution has been found to be 30 points per unit nondimensional length. Accordingly a mesh with 600 points in the $\mathrm{x}$ direction and 30 points in the y direction has been used for all simulations (i.e. 30 grid points per unit nondimensional length along both spatial horizontal and vertical directions, see Table I). The criterion for the selection of the temporal resolution (i.e. the time integration step) has been based on the combination of three independent requirements:

$$
\begin{aligned}
& \Delta t_{1} \leq \frac{1}{2 \operatorname{Pr}} \frac{\Delta x^{2} \Delta y^{2}}{\left(\Delta x^{2}+\Delta y^{2}\right)} \\
& \Delta t_{2} \leq \frac{1}{2} \frac{\Delta x \Delta y}{\left(u_{\max } \Delta y+v_{\max } \Delta x\right)} \\
& \Delta t_{3} \leq \frac{2 \pi}{\varpi M} \text { where } \mathrm{M}=1000
\end{aligned}
$$




$$
\Delta t=\min \left\{\Delta t_{1}, \Delta t_{2}, \Delta t_{3}\right\}
$$

where the first two inequalities are the well-known conditions for the numerical stability of the diffusive and convective contributions, respectively, while the third one is an even more restrictive limitation coming from the need to "resolve" with a sufficient number $\mathrm{M}$ of time steps (a minimum of $10^{3}$ ) the period of oscillation of the imposed vibrations. The first condition depends on the spatial resolution only. The second has been found to be the dominant limitation at high values of the Rayleigh number $\mathrm{Ra}_{\omega}$ defined by eq. (1b), whereas the third becomes effective only at relatively high values of the angular frequency $\left(\varpi=10^{4}\right)$.

\section{G. Validation}

Validation of the numerical algorithm has been attained via comparison with the numerical results by Shevtsova et al. [17] for the case of mixed thermocapillary-thermogravitational convection (steady gravity). Their numerical results were obtained for values of the characteristic parameters relatively close to those considered here $(A=24.7, \operatorname{Pr}=13.9)$. In particular we refer to their simulations for $\mathrm{Ma}=18154.5$ and $\mathrm{Ra}=2578\left(\mathrm{Bo}_{\mathrm{dyn}}=\mathrm{Ra} / \mathrm{Ma}=0.142\right.$, Grid $\left.600 x 100\right)$ for which they could clearly measure the frequency of the emerging traveling wave as $\Omega_{\mathrm{HTW}}=45.28$. By using such values of the aspect ratio, Prandtl number and Marangoni number, and simply setting $\varpi=0$ and $\mathrm{Ra}_{\omega}=2578$ in our code (with a mesh $600 \mathrm{x} 30$ ), we could obtain a frequency 46.03 differing by $1.6 \%$ with respect to the value reported in their work.

\section{Results}

We concentrate on the following specific conditions: fixed values for the aspect ratio and Prandtl and Marangoni numbers $\left(\mathrm{A}=20, \mathrm{Pr}=15, \mathrm{Ma}=3 \times 10^{4}\right)$, the nondimensional angular frequency of the acceleration disturbance and the associated Rayleigh number spanning over several orders of magnitude. More precisely, $\varpi$ is allowed to vary in the interval $\Omega_{\mathrm{HTW}} \leq \varpi \leq 1 \times 10^{4}$ (where $\Omega_{\mathrm{HTW}}$ is the angular frequency of the hydrothermal wave produced by pure Marangoni flow), and the Rayleigh number spans the range $0 \leq \mathrm{Ra}_{\omega} \leq 3 \times 10^{6}$. By introducing a specific parameter measuring the relative importance of vibrational and Marangoni effects as:

$$
B_{\omega}=\frac{b \omega^{2} \rho \beta_{T}}{\sigma_{T}} L^{2}=\frac{R a_{\omega}}{M a}
$$

the last condition is equivalent to $0 \leq \mathrm{B}_{\omega} \leq 10^{2}$.

More specifically, for each interval $10^{\mathrm{n}-1} \leq \mathrm{Ra}_{\omega}<10^{\mathrm{n}}$ with $4 \leq \mathrm{n} \leq 7$ and $10^{\mathrm{n}-1} \leq \boldsymbol{\omega}<10^{\mathrm{n}}$ with $2 \leq \mathrm{n} \leq 5$, numerical simulations have been performed for $\mathrm{Ra}_{\omega}=\mathrm{a} 10^{\mathrm{n}-1}$ and $\varpi=\mathrm{b} 10^{\mathrm{n}-1}$ considering for a values $1,3,5$ and 10 and for $b 1$ and 5, respectively. This resulted in a total of approximately 160 simulations. 
Moreover, we limited ourselves to considering two-dimensional flow having in mind that such an idealized setting will help us in using it to infer the general principles which drive complex patterns in more realistic situations (in which vibrations and Marangoni flow can also display components along the third dimension, the subject of a companion paper).

\section{A. Pure Marangoni flow and the Classical Hydrothermal wave}

To fully understand the influence of inertial (vibrational) effects on the supercritical state of Marangoni flow, we begin our analysis from the simplest possible situation, i.e. that of unperturbed (vibration-less) thermocapillary convection. It is known (see, e.g., [30]) that for this case, the socalled "hydrothermal waves" (HTW) always correspond to the preferred mode of instability (Smith and Davis [36]). These wave are known to have weak components in the flow spanwise direction in the high-Pr case [37-39], which supports our assumption of two-dimensional flow. A remarkable feature common to all cases is that the disturbance always travels in a direction with a component in the direction opposite to that of the surface flow (upstream).

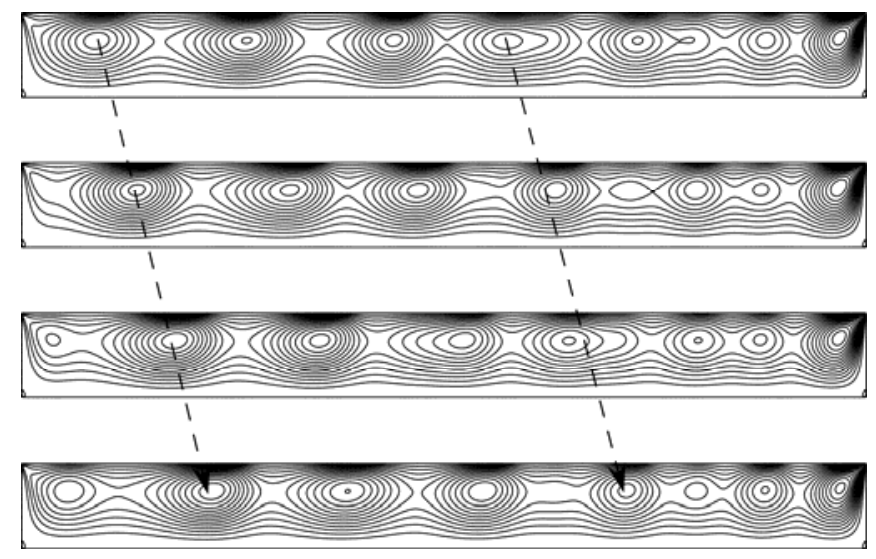

Figure 2: Oscillatory instability of Marangoni flow in a liquid layer $\left(\operatorname{Pr} \cong 15, A=20, M a=3 \times 10^{4}\right.$, Ma based on the depth; adiabatic free surface; cold side on the left, hot side on the right; the isolines of the stream-function $\left(\psi_{\max }=43.3, \Delta \psi \cong 3.1\right)$ are shown in four snapshots (plane $(\mathrm{x}, \mathrm{y})$ ) evenly distributed during one period of oscillation $\tau_{\mathrm{HTW}}=2 \pi / \Omega_{\mathrm{HTW}}$ ): The location of the cells near the cold side at different time moments indicates the propagation of a wave to the right, i.e. in the upstream direction; a single roll is steadily located near the hot wall as a consequence of the strong temperature gradient established in the boundary layer adjacent to the right wall (for illustration purposes, in the figure above the depth of the fluid layer is two times its real dimension).

As shown in Fig. 2, in practice, the hydrothermal wave itself looks as a chain of cells moving from the cold side towards a motionless rolls on the hot side with a given angular frequency $\left(\Omega_{\mathrm{HTw}}=\right.$ 47.1 for $\mathrm{A}=20, \mathrm{Pr}=15, \mathrm{Ma}=3 \times 10^{4}$ ). The stationary roll existing near the right wall is maintained by the strong temperature gradient established in the lateral boundary layer. The moving train of moving cells "feels" the presence of this roll by decaying in the region where this roll is located. When a roll "dies" in proximity to the hot side, a new roll is created at the cold side thereby 
preserving the average number $\mathrm{N}$ of convective cells present in the cavity at any instant ( $N \cong 7$ for the present conditions).

\section{B. The thermovibrational flow and its properties}

Before embarking into the systematic analysis of hybrid thermocapillary-thermovibrational convection, following the same practice undertaken in Sect. IIIA for Marangoni flow, we recall here some fundamental properties of pure thermovibrational convection (which will prove very useful in the interpretation and categorization of mixed flows considered later).

It is known (see, e.g., $[2,10]$ ) that in the presence of an imposed acceleration disturbance of given angular frequency $\omega$, the velocity field $\underline{V}$ will be, in general, made up by the sum of two contributions, an average value $\underline{\bar{V}}$ plus a periodic oscillation of amplitude $\underline{V}^{\prime}\left(\underline{V}^{\prime}=\underline{V}-\underline{\bar{V}}\right)$ oscillating in time at the same acceleration frequency $\omega$ of the imposed disturbance (or at frequencies that are multiple of $\omega$ ). In the absence of pre-existing flows of other natures, this average (steady) contribution $\underline{\bar{V}}$ should be regarded essentially as a consequence of the non-linear nature of the balance equations, which (by virtue of such a non-linearity) are able to produce a flow that has a non-zero finite steady component although the imposed alternating force does not possess such a property.

The same concept applies to any scalar field (e.g. the temperature) associated with the considered convective field. As a result of such a convective field, the scalar variables can be also thought of as quantities consisting of a steady plus an oscillatory part $\left(T=\bar{T}+T^{\prime}\right)$.

The relative "weight" of the two contributions, in general, is a part of the problem, i.e. it cannot be predicted "a priori"; different situations may occur, depending on the oscillation frequency. For instance, it is known that at high frequencies a regime can be attained where the oscillatory velocity and temperature contributions are relatively large while the related time-average steady contributions are small. At smaller frequencies, the situation is reversed, with the oscillatory contributions being very strong with respect to the mean ones.

\section{The High frequency regime}

When vibrations are superimposed on an already developed convective flow (of thermocapillary nature in our case) the situation becomes even more complex in terms of system response and related non-linearities. The effect of vibrations strongly depends on the shaking direction relative to the prevailing temperature gradient. In particular, it is known that, in the limit of high frequency $(\varpi)$ and small displacement ( $\Lambda=b \frac{\beta_{T} \Delta T}{L}$ ) (see Birikh at al. [19]), the mean vibration force is a bulk driving action induced by temperature gradients normal to the vibration axis, an important feature of this force being that, if temperature distortions, with respect to the purely diffusive case, are 
induced by another type of convection, average vibrational flows arise in such a way as to permit the isotherms to turn and again become perpendicular to the vibration direction.

Accordingly, vibrations are expected to exert a damping action on pre-existing Marangoni convection if applied in the same direction of the imposed temperature gradient (along the liquid/gas interface in the present case).

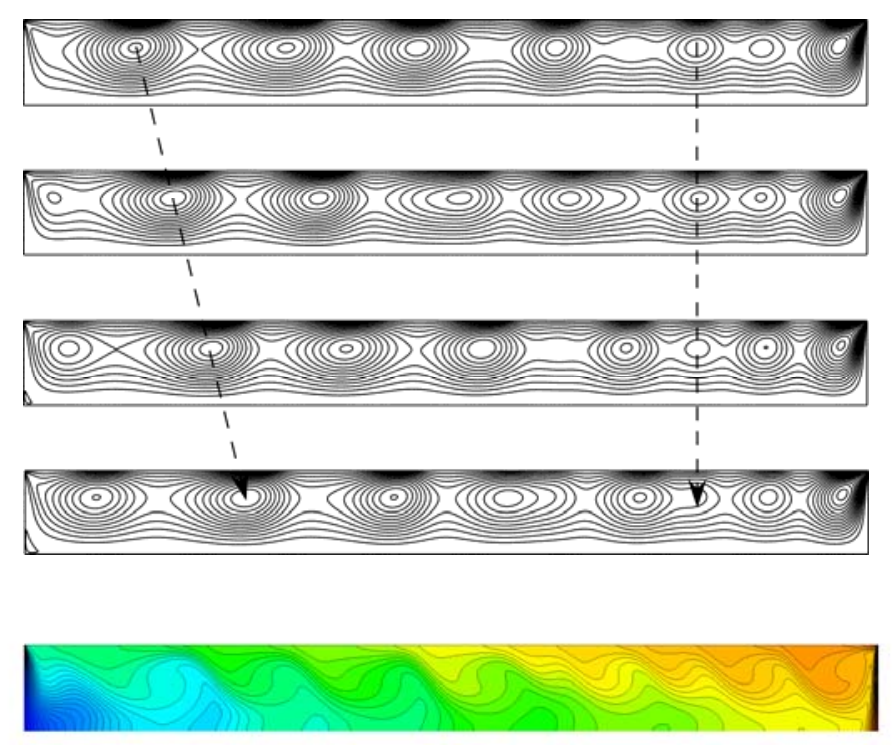

Figure 3: Mixed Marangoni-vibrational flow $\left(\mathrm{Ma}=3 \times 10^{4}, \varpi=10^{4}, \mathrm{Ra}_{\omega}=3 \times 10^{4}, \mathrm{~B}_{\omega}=\mathrm{Ra}_{\omega} / \mathrm{Ma}=1, \theta=0\right.$, vibrations parallel to liquid/gas interface, $\psi_{\max }=43.75, \Delta \psi \cong 3.1$, streamlines are shown in four snapshots evenly distributed during the timeframe $\tau_{\mathrm{HTW}}$. The additional bottom contour map shows a snapshot of the related temperature field.

Some results along these lines are shown in Fig. 3 which illustrates the stages of evolution of supercritical Marangoni flow in the presence of imposed vibrations with $\varpi=10^{4}$ and $\operatorname{Ra}_{\omega}=3 \times 10^{4}$ $\left(\mathrm{B}_{\omega}=1, \theta=0\right)$.

As expected, some mitigation of the typical features of the hydrothermal wave can be observed. Indeed, this Figure shows that the velocity of propagation of rolls changes along the cavity, as the rolls can be clearly seen to decelerate in the course of their migration from the cold side towards the hot side. Their velocity decreases until it vanishes completely at a certain location; there the typical spatiotemporal behaviour of the traveling wave is taken over by a different mechanism by which rolls occupy fixed positions in time.

Most interestingly, the resulting flow pattern can be ideally split into two different regions:

A group of cells spatially spreading periodically towards the hot side (i.e. moving upstream), represents a first circulation system. Such a group of rolls is bounded from the left (where such rolls are being continuously created) by the cold wall and from the right (where their propagation velocity decays) by a second multicellular region where convection displays a quasi-steady behaviour. This region is characterized by a relatively stable pattern consisting of corotating quasi- 
stationary rolls. Although these rolls undergo some weak growth and decay in time, they maintain stable positions (thereby, creating a 'barrier', i.e. a resistance to the propagation of the rolls being continuously created at the cold side and spreading towards the hot side).

Interestingly, due to the impact of the moving rolls coming from the cold side, an intermediate region is created where the local patterning behaviour consists of the alternance of two distinct corotating rolls and a single vortex formed by the periodic merging of such rolls. As a result of the co-existence of three different regions, the number of convective rolls present at any instant in the layer increases with respect to the case of pure Marangoni flow ( $\mathrm{N}=8$ for the present conditions).

Most interestingly, Fig. 4 shows that if the considered Rayleigh number is increased by one order of magnitude $\left(\operatorname{Ra}_{\omega}=3 \times 10^{5}\right)$ while retaining the same frequency of shaking $\left(\varpi=10^{4}\right)$, the multicellular flow where convection displays a quasi-steady behaviour becomes the dominant mode of convection (it extends to the entire layer). The number of rolls present at any time in the layer increases from a total of 8 (for $\mathrm{Ra}_{\omega}=3 \times 10^{4}$ ) to $\mathrm{N}=10$. Cells spatially spreading periodically towards the hot side are no longer a feature of the system. A second set of cells, however, appears periodically in proximity to the bottom wall (Figure 4b). Such cells have a very limited extension along the y direction and display a clockwise sense of circulation (i.e. they may be seen as a counter-rotating system of circulation with respect to the classical Marangoni flow).
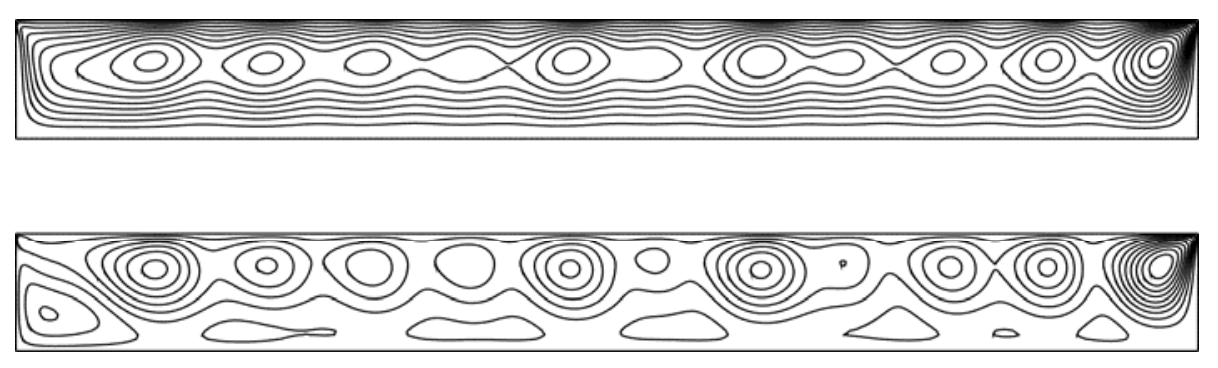

Figure 4: Mixed Marangoni-vibrational flow $\left(\mathrm{Ma}=3 \times 10^{4}, \varpi=10^{4}, \mathrm{Ra}_{\omega}=3 \times 10^{5}, \mathrm{~B}_{\omega}=\mathrm{Ra}_{\omega} / \mathrm{Ma}=10\right.$, $\theta=0$, vibrations parallel to liquid/gas interface, $\psi_{\min }=-9.5, \psi_{\max }=50.5, \Delta \psi \cong 4.3$, streamlines are shown in two snapshots).

When vibrations are rotated by $90^{\circ}$ (resulting oscillatory acceleration perpendicular to the imposed temperature gradient), in line with the general expectations related to the Birikh's principle, we found changes induced in the HTW to be barely appreciable for $\mathrm{Ra}_{\omega}=3 \times 10^{4}$. For this case the qualitative mechanism with cells migrating continuously in the upstream direction is retained, although for larger values of $\mathrm{Ra}_{\omega}$ some wave "modulation" effects due to the application of vibrations become noticeable. 


\section{Gravitationally modulated HTWs at intermediate frequencies}

As already discussed to a certain extent in Sect IIIB, there is a strong dependence of both types of vibrations-induced contributions (average and periodic) on the acceleration frequency. Amplitudes of the periodic (oscillatory) contributions tend quickly to increase with a decrease in the forcing frequency. Conversely the average quantities are less dependent on $\varpi$ so that at low frequencies one may expect the steady contribution to become negligible in comparison with the unsteady one. As a result, the general Birikh's law discussed in Sect IIIC (about the expected effect of the mean vibration force) is no longer applicable at low and intermediate frequencies and the influence of vibrations on supercritical Marangoni flow becomes essentially unpredictable (i.e. it has to be assessed on a case-by-case basis).

As a first step towards this end, numerical simulations performed yet for $\operatorname{Ra}_{\omega}=3 \times 10^{4}$ and $\theta=0$, but decreasing the angular frequency by one order of magnitude (i.e. $\varpi=10^{3}$ ), reveal that vibrations directed along the liquid/gas interface are no longer able to induce the changes (in terms of patterning behaviour and spatio-temporal dynamics) observed for $\varpi=10^{4}$. Indeed, apart from some weak modulation of the rolls intensity, the velocity field (not shown) does not display significant changes with respect to the case of pure thermocapillary flow (the reader being referred again to Fig. 2, which shows rolls continuously propagating in the upstream direction).

At this frequency, the order of magnitude of the Rayleigh number has to be increased to produce a significant effect. As an example, Fig. 5, illustrates the convective field for $\varpi=10^{3}, \mathrm{Ra}_{\omega}=3 \times 10^{5}$ in the case of vibrations parallel to the liquid/gas interface. For this value of the Rayleigh number, the travelling wave is completely replaced by a new mechanism with rolls at fixed locations which "pulsate" in time. (i.e. disturbance nodes growing and shrinking alternately in time without undergoing an appreciable displacement in the streamwise direction). A more-in-depth analysis of Figure 5 also reveals that the classical dynamics seen in Fig. 2 (pure Marangoni flow) and Fig. 3 $\left(\varpi=10^{4}, \mathrm{Ra}_{\omega}=3 \times 10^{4}\right)$, characterized by convective rolls all rotating in the anticlockwise direction (and all being part of a larger circulation system with fluid moving continuously from the hot side to the cold side along the free interface and then flowing back along the bottom wall), is no longer a feature of the flow. Regions of reversed flow, in fact, appear periodically at the free interface, which correspond to the emergence of clockwise-oriented cells alternatively distributed among the anticlockwise-oriented rolls (see, e.g., frames (g-h)) as witnessed by the negative value of the streamfunction associated with them. Accordingly, the number of rolls present in the layer at any instant ranges between $\mathrm{N}=9$ (when all rolls have the same sense of circulation, see, e.g., frames (be)) and $\mathrm{N}=16$ (see frames (g-h), where the flow may be seen as a set of 8 couples of counterrotating cells). 

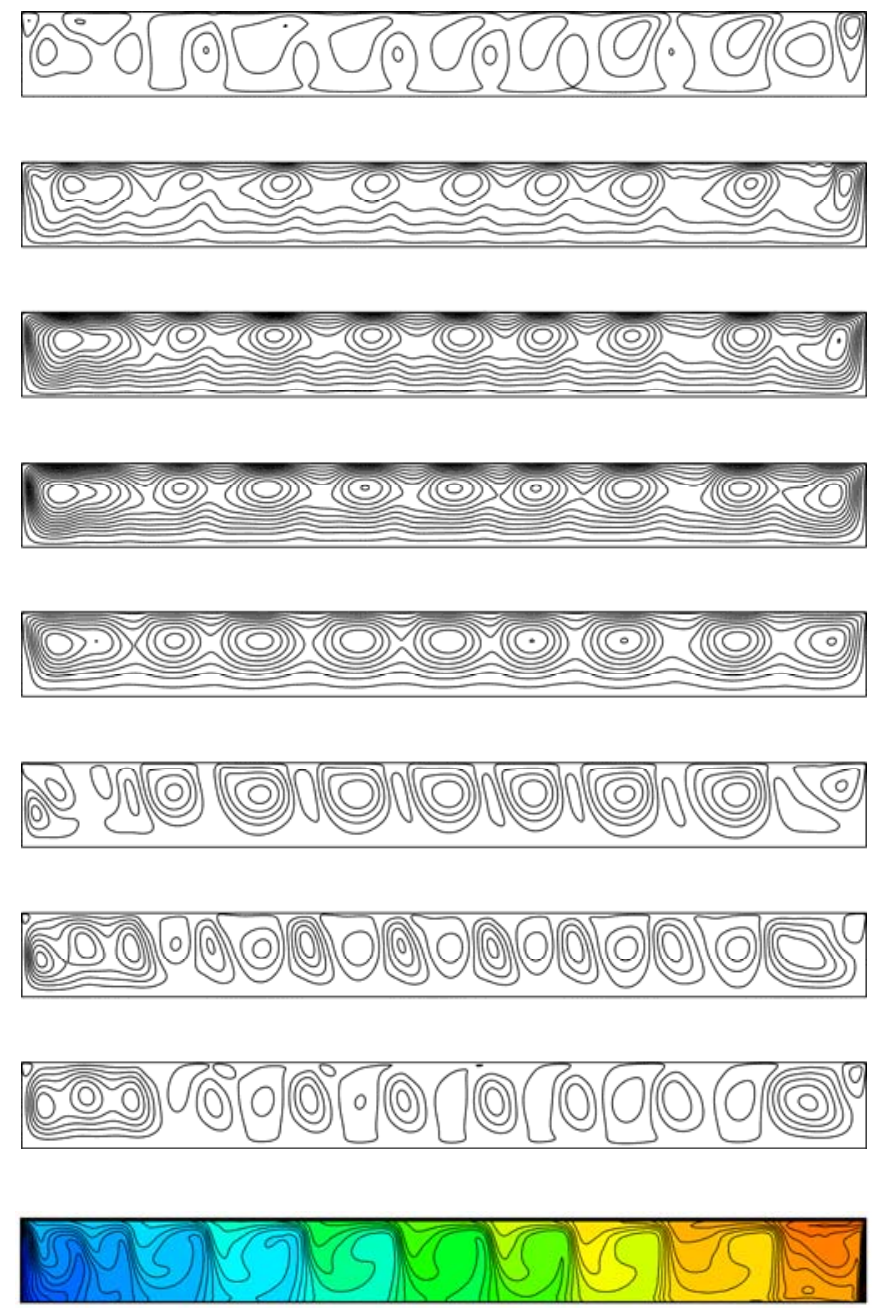

Figure 5: Mixed Marangoni-vibrational flow $\left(\mathrm{Ma}=3 \times 10^{4}, \varpi=10^{3}, \mathrm{Ra}_{\omega}=3 \times 10^{5}, \mathrm{~B}_{\omega}=\mathrm{Ra}_{\omega} / \mathrm{Ma}=10\right.$, $\theta=0$, vibrations parallel to liquid/gas interface, $\psi_{\min }=-55.5, \psi_{\max }=99.2, \Delta \psi \cong 8.14$, streamlines are shown in eight snapshots evenly distributed during the timeframe $\tau_{\omega}=2 \pi / \varpi$ ). The additional bottom contour map shows a snapshot of the related temperature field.

In the following we will classify the emerging solutions as: travelling (TR) and standing (SR) rolls solutions (or as travelling (TW) and standing (SW) waves, respectively) depending on whether the prevailing oscillatory mode is featured by disturbance nodes propagating along the $\mathrm{x}$ direction, or growing and shrinking alternately in time at fixed positions. 

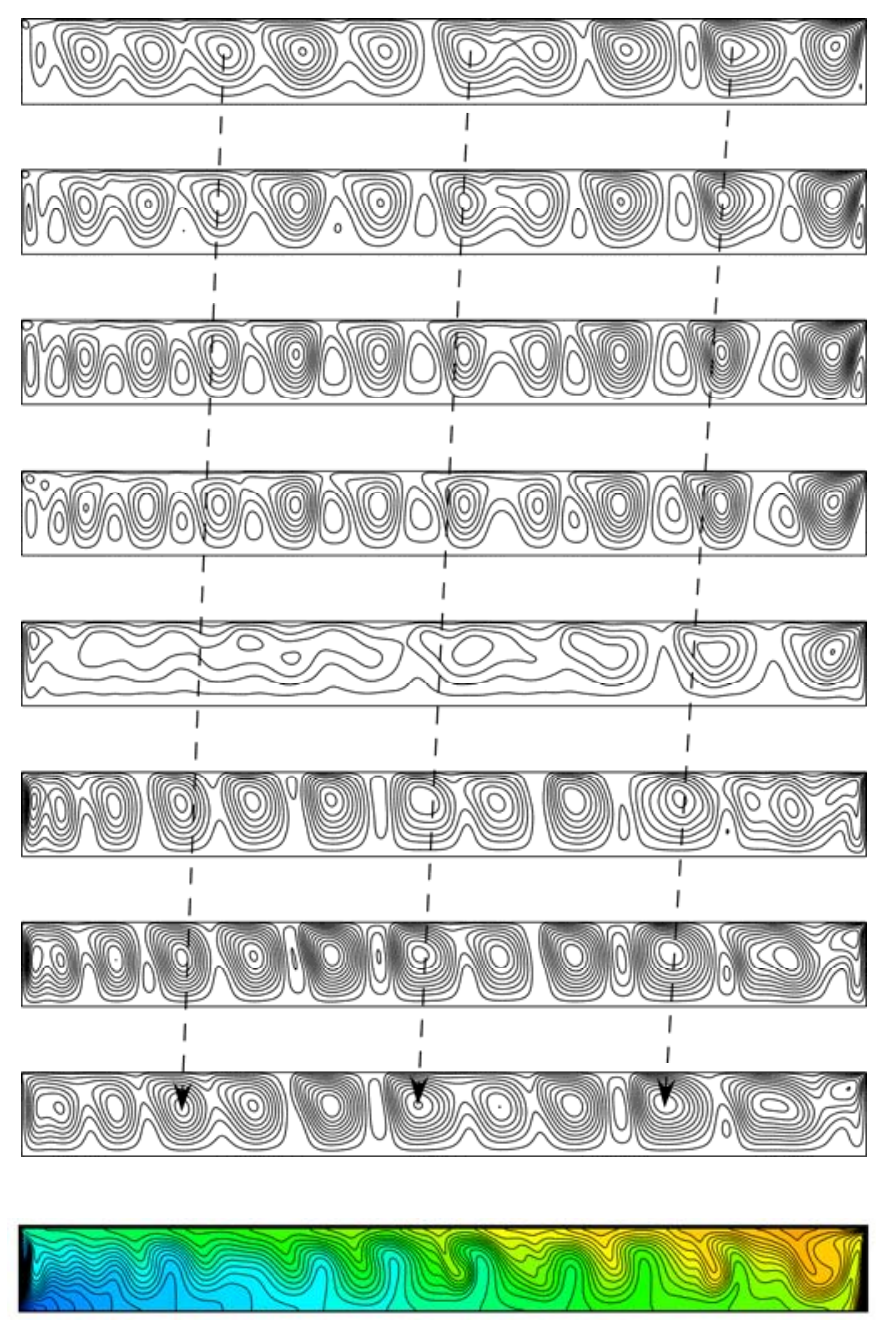

Figure 6: : Mixed Marangoni-vibrational flow $\left(\mathrm{Ma}=3 \times 10^{4}, \varpi=10^{3}, \mathrm{Ra}_{\omega}=3 \times 10^{5}, \mathrm{~B}_{\omega}=\mathrm{Ra}_{\omega} / \mathrm{Ma}=10\right.$, $\theta=\pi / 2$, vibrations perpendicular to liquid/gas interface, $\psi_{\min }=-26.5, \psi_{\max }=76.1, \Delta \psi \cong 7.3$ streamlines are shown in eight snapshots evenly distributed during the timeframe $\tau_{\text {HTW }},|\Omega| \cong 420$ ). The additional bottom contour map shows a snapshot of the related temperature field.

Most notably, an even more dramatic change in the emerging dynamics with respect to those obtained for $\varpi=10^{4}$ can be produced when the direction of vibrations is changed to make them perpendicular to the liquid/gas interface $\left(\varpi=10^{3}, \operatorname{Ra}_{\omega}=3 \times 10^{5}, \theta=\pi / 2\right)$.

Figure 6 clearly indicates that for such circumstances the cells spatially spread from the hot side towards the cold side (i.e. they move downstream). This means the nodes of the gravitationally modulated wave have reversed their direction of propagation with respect to the vibration-less condition (recall pure hydrothermal waves propagate in the upstream direction as extensively discussed in the preceding text). This may be seen as a first practical occurrence of the principle by which the application of a proper modulation strategy may be used to induce a desired spatiotemporal change resulting in disturbance nodes travelling in the positive (upstream) or negative (downstream) direction, or not travelling at all (a standing wave). 
Most interestingly, when the wave reverses its sense of propagation, the related angular frequency ( $\Omega \mid \cong 420$ ) is much larger than the typical angular frequency of the HTW ( $\Omega \cong 47.1$ ), which clearly indicates a significant modification of the fundamental mechanism of instability.

Like the case of vibrations parallel to the interface, also for $\theta=\pi / 2$, clockwise-oriented rolls separating anticlockwise rotating cells emerge. In this case, however, as noticeable in Fig. 6, their longitudinal (along $\mathrm{x}$ ) size is very limited (it never reaches the same size of anticlockwise-oriented cells). On average the number of anticlockwise rolls is $\mathrm{N}=9$.

Interestingly, an analysis of the associated temperature field reveals the existence of 10 thermal plumes originating from the bottom wall and extending towards the free surface, resembling those that would be typical of a classical layer heated from below and subjected to standard gravity (Rayleigh-Bénard convection). It can be therefore argued that the mechanism driving instability has a strong component of buoyancy nature (i.e. related to the inertial action produced by vibrations; the reader is referred to Sect. IV for some additional insights into these dynamics).
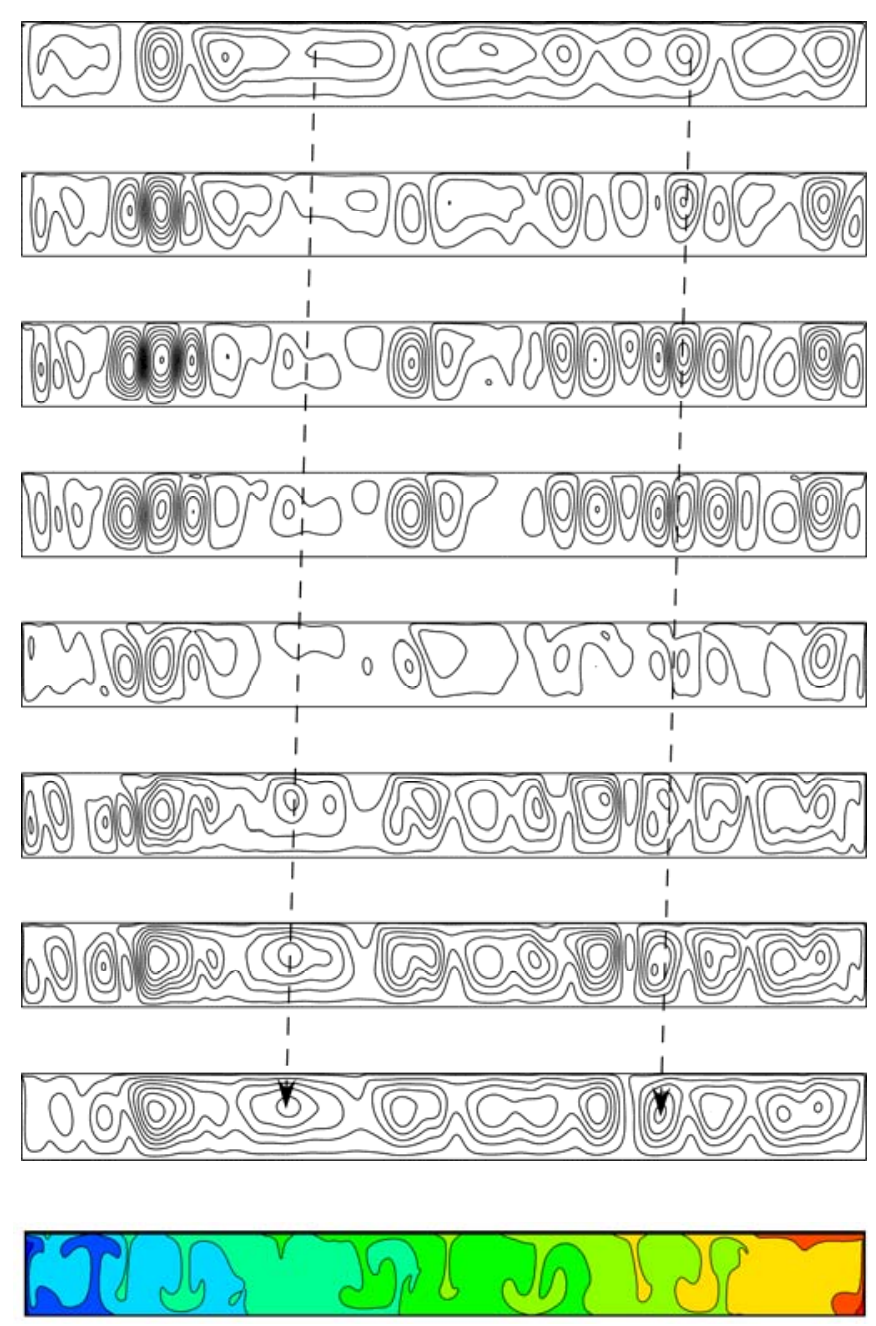

Figure 7: Marangoni-vibrational flow $\left(\mathrm{Ma}=3 \times 10^{4}, \varpi=10^{3}, \mathrm{Ra}_{\omega}=1 \times 10^{6}, \mathrm{~B}_{\omega}=\mathrm{Ra}_{\omega} / \mathrm{Ma}=33, \theta=\pi / 2\right.$, vibrations perpendicular to liquid/gas interface, $\psi_{\min }=-169.5, \psi_{\max }=178.5, \Delta \psi \cong 24.85$, streamlines are shown in eight snapshots evenly distributed during the timeframe $\left.\tau_{\omega}=2 \pi / \varpi,|\Omega| \cong 193\right)$. The additional bottom contour map shows a snapshot of the related temperature field. 
An increase in the Rayleigh number $\left(\operatorname{Ra}_{\omega}=1 \times 10^{6}, \theta=\pi / 2\right.$, Fig. 7) determines an increase in size of the clockwise-oriented rolls. Figure 7 , showing the typical sequence of stages evolution in the period $2 \pi / \varpi$, indicates that although counterclockwise rolls are the dominant mode of convection in frames (f-h), in the other stages of evolution they have the same size as the clockwise-oriented ones (as also confirmed by $\psi_{\min }$ and $\psi_{\max }$ whose absolute values are almost equal). Moreover, comparison of frames (a) and (h) leads to the conclusion that the direction of propagation of convective disturbances is still in the downstream direction. The related angular frequency, however, experiences a decrease with respect to the value measured for $\operatorname{Ra}_{\omega}=3 \times 10^{5}$.

An additional increase in the Rayleigh number further reduces the velocity of the disturbances, which drops from -420 for $\mathrm{Ra}_{\omega}=3 \times 10^{5}$ to -193 for $\mathrm{Ra}_{\omega}=1 \times 10^{6}$ to $\Omega \cong-48$ only for $\mathrm{Ra}_{\omega}=3 \times 10^{6}$ (Figure 8).

In line with the expectations naturally resulting from the observed decrease of the disturbance angular velocity for increasing values of the Rayleigh number, only standing waves have been obtained for values of the Rayleigh number higher than $3 \times 10^{6}$.

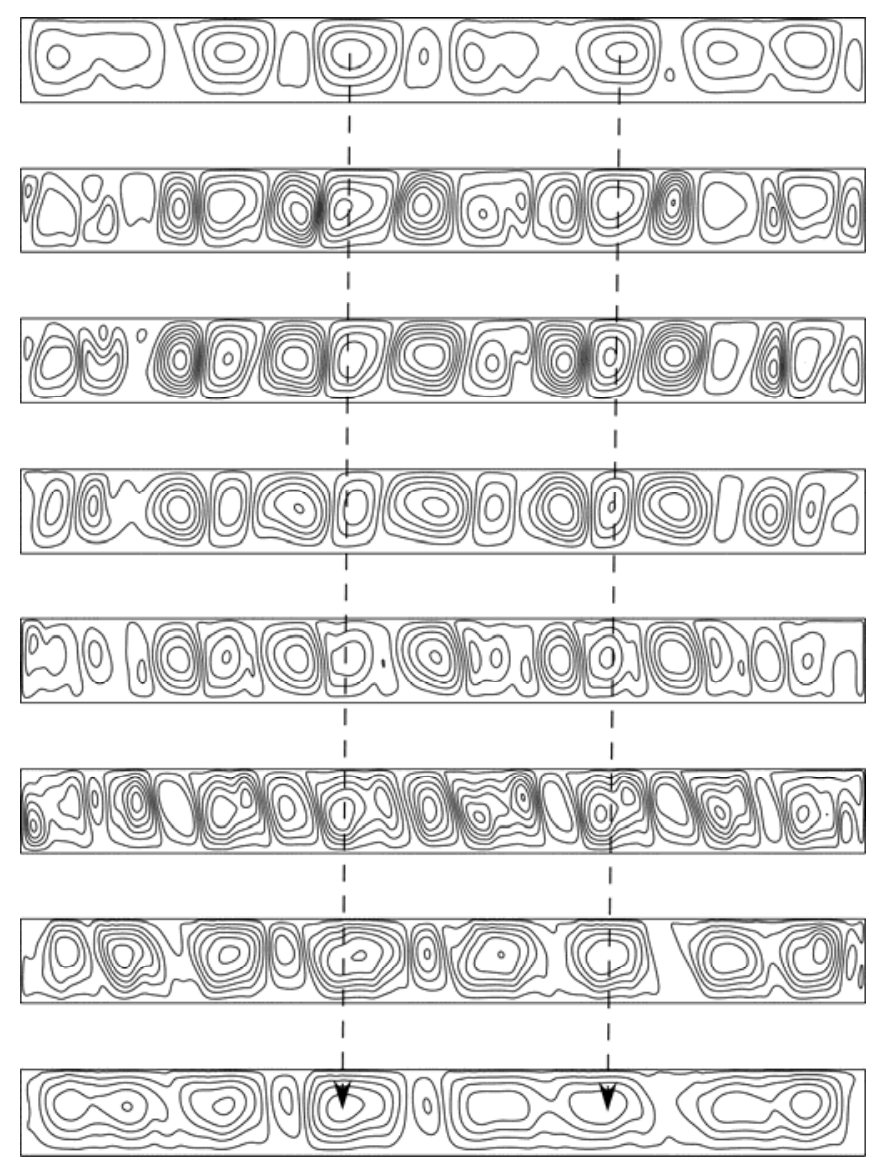

Figure 8: Marangoni-vibrational flow $\left(M a=3 \times 10^{4}, \varpi=10^{3}, \mathrm{Ra}_{\omega}=3 \times 10^{6}, \mathrm{~B}_{\omega}=\mathrm{Ra}_{\omega} / \mathrm{Ma}=10^{2}, \theta=\pi / 2\right.$, vibrations perpendicular to liquid/gas interface, $\psi_{\min }=-415.8, \psi_{\max }=340, \Delta \psi \cong 54$, streamlines are shown in eight snapshots evenly distributed during the timeframe $\tau_{\omega}=2 \pi / \varpi,|\Omega|=48$ ). 
A further decrease in the frequency of forcing $\left(\varpi=10^{2}\right)$ confirms the trend discussed in the preceding text about the relative importance of vibration-induced steady (averaged) and oscillatory velocity (and temperature) contributions. In such conditions the time-averaged departure from the basic solution induced by the vibrational effect is very small with respect to the oscillatory component of such a departure. The related effects involve significant temporal modifications to the earlier flow solutions (the supercritical Marangoni flow), which depend basically on the intensity of the forcing (the Rayleigh number $\mathrm{Ra}_{\omega}$ ) and its direction.

In particular, vibrations in the vertical direction $\left(\theta=90^{\circ}\right)$ are expected to generate time-dependent vorticity due to coupling with the applied horizontal temperature gradient (this alternately cooperating or competing with the Marangoni flow over a cycle of the modulation, Grassia and Homsy [21]), while vibrations applied along the layer $\left(\theta=0^{\circ}\right)$ to produce vorticity only when coupled to vertical convected temperature gradients (produced by the pre-existing Marangoni flow). Present numerical simulations for $\varpi=10^{2}$ indicate that, by virtue of mechanisms depicted above, the flow at $\operatorname{Ra}_{\omega}=3 \times 10^{5}$ is rather turbulent for both $\theta=0^{\circ}$ and $\theta=90^{\circ}$ (not shown) in comparison to earlier cases at higher values of $\varpi$.
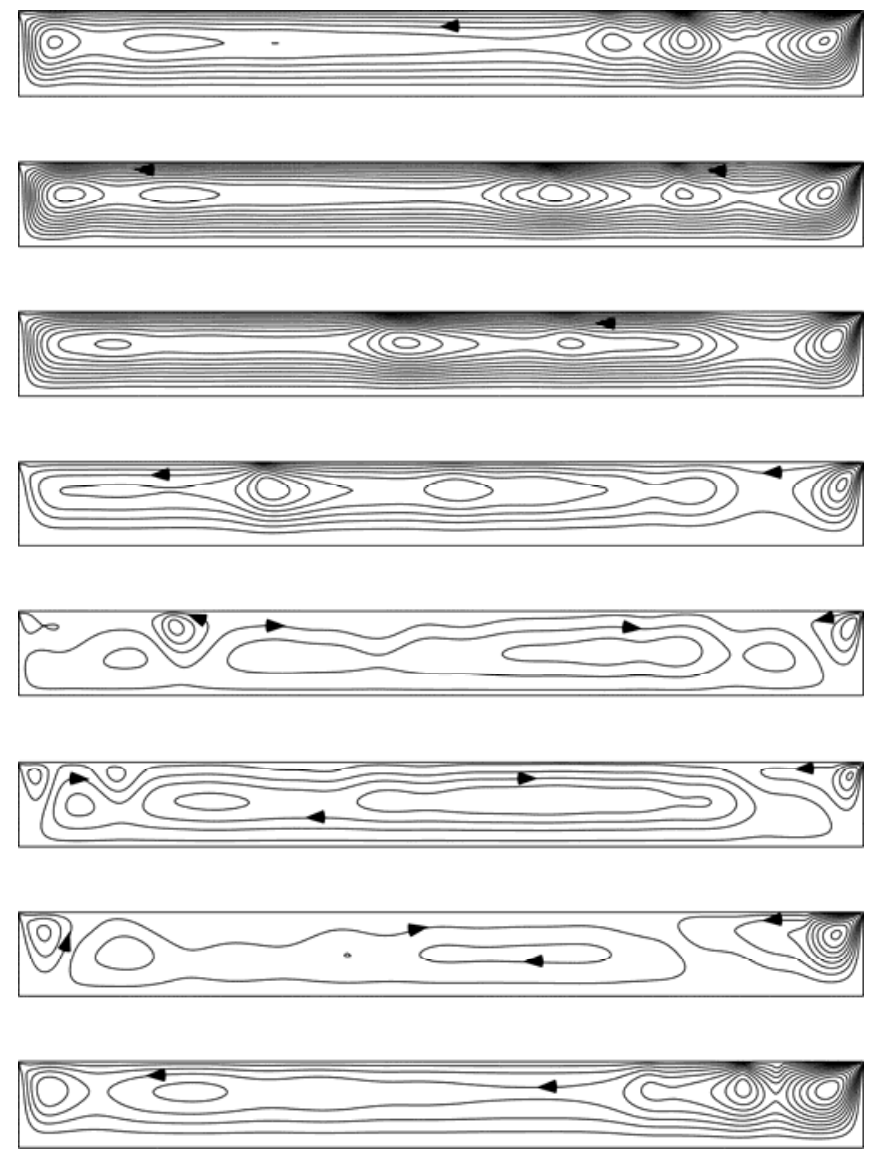

Figure 9: Mixed Marangoni-vibrational flow $\left(\mathrm{Ma}=3 \times 10^{4}, \omega=10^{2}, \mathrm{Ra}_{\omega}=3 \times 10^{4}, \mathrm{~B}_{\omega}=\mathrm{Ra}_{\omega} / \mathrm{Ma}=1, \theta=0\right.$, vibrations parallel to liquid/gas interface, $\psi_{\min }=-23.9, \psi_{\max }=73.5, \Delta \psi \cong 5.73$, streamlines are shown in eight snapshots evenly distributed during the timeframe $\left.\tau_{\omega}=2 \pi / \varpi\right)$. 
Interestingly, for $\theta=0^{\circ}$ and $\operatorname{Ra}_{\omega}=3 \times 10^{4}$ (see Figure 9), the interplay between the imposed horizontal vibrations over a cycle of the modulation and temperature gradients produced by Marangoni flow leads to a periodic response consisting of the temporal alternance of two main circulation systems. One of them is represented by a counterclockwise oriented elongated cell where fluid moves continuously on the free surface from the hot side towards the cold side (and then back to the hot side along the bottom wall). The other is a more complex configuration in which an extended region of reversed flow appears at the free surface. This region corresponds to the presence of an elongated (70 \% of the overall system horizontal size, see, e.g., frames (e)-(g)) single convective cell rotating in the clockwise direction, while the remaining part of the layer is occupied by two rolls of lower size still rotating in the anticlockwise sense (located in proximity to the lateral walls).

The former circulation system can be seen as a result of the joint action of the Marangoni effect and the Rayleigh-Bénard convective mode that is excited when the system is temporarily subjected to an acceleration acting along the positive $\mathrm{x}$ direction (i.e. in the part of the cycle when the stratification induced along the $\mathrm{x}$ direction by the imposed temperature gradient is unstable with respect to the acceleration). In this configuration the body force, which acts to displace the hot light fluid by the cold heavy fluid, tends to enhance the basic Marangoni return flow.

Figure 9 also shows that the multicellular character of convective systems (with many rolls) seen for higher values of $\varpi$ is significantly reduced in such circumstances. This clearly indicates that the oscillatory nature of the thermofluid-dynamics field and the related large-scale disturbances must be ascribed essentially to the imposed forcing rather than to the existence and propagation of a hydrothermal wave of thermocapillary origin.

When vibrations perpendicular to the liquid surface are considered (see Figure $10, \theta=90^{\circ}$ and $\mathrm{Ra}_{\omega}=3 \times 10^{4}$ ), comparison with the earlier simulations performed for $\varpi=10^{3}$ and $\mathrm{Ra}_{\omega}=3 \times 10^{5}$, makes it immediately evident that a Rayleigh number decreased by one order of magnitude is no longer sufficient to support the reversal of direction of propagation of the wave seen in Figure 6. 


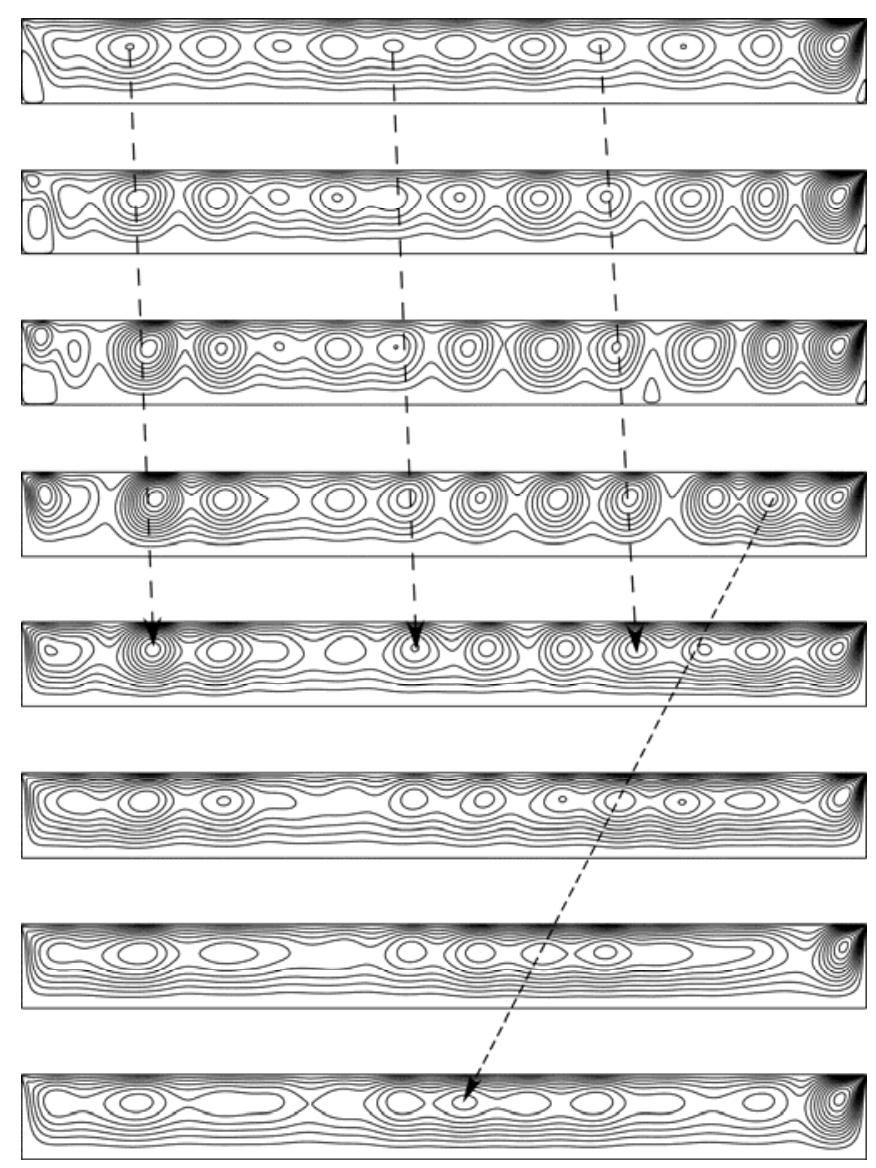

Figure 10: Mixed Marangoni-vibrational flow $\left(\mathrm{Ma}=3 \times 10^{4}, \varpi=10^{2}, \mathrm{Ra}_{\omega}=3 \times 10^{4}, \mathrm{~B}_{\omega}=\mathrm{Ra}_{\omega} / \mathrm{Ma}=1\right.$, $\theta=\pi / 2$, vibrations perpendicular to liquid/gas interface, $\psi_{\min }=-8, \psi_{\max }=47.8, \Delta \psi \cong 4$, streamlines are shown in eight snapshots evenly distributed during the timeframe $\tau_{\omega}=2 \pi / \varpi$ ).

The rolls in Figure 10, in fact, display again the classical motion in upstream direction which is typical of the canonical Marangoni supercritical flow (the reader is referred in particular to the left part of the figure). Nevertheless a large-scale disturbance traveling in the same direction of the surface flow (downstream) can be still identified. Originating from the hot side it propagates on the background flow causing a visible weakening of the rolls. Under the impact of such a disturbance, any roll belonging to the Marangoni multicellular structure is weakened for a limited time until the disturbance moves to the next roll in the downstream direction. Such a disturbance travels with the angular velocity of the forcing acceleration (๗). 


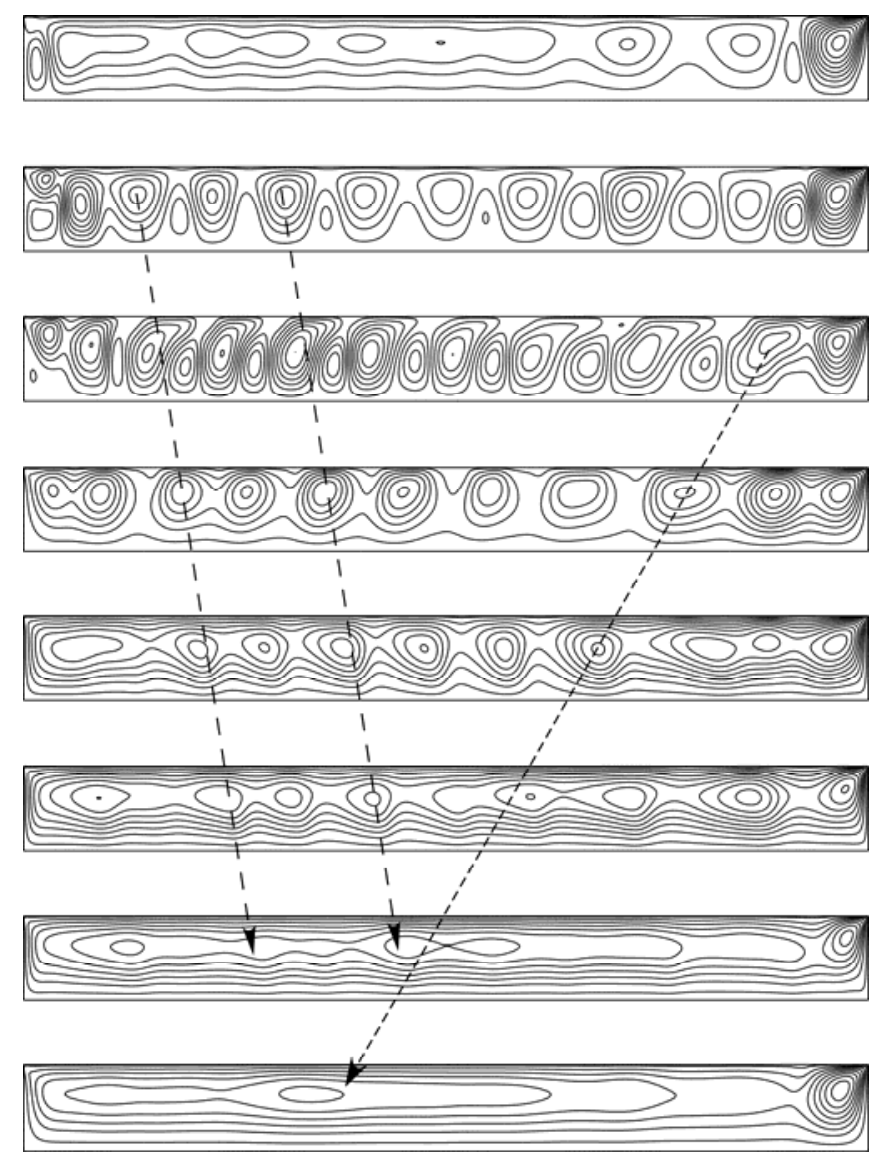

Figure 11: Mixed Marangoni-vibrational flow $\left(\mathrm{Ma}=3 \times 10^{4}, \varpi=10^{2}, \mathrm{Ra}_{\omega}=1 \times 10^{5}, \mathrm{~B}_{\omega}=\mathrm{Ra}_{\omega} / \mathrm{Ma}=3.3\right.$, $\theta=\pi / 2$, vibrations perpendicular to liquid/gas interface, $\psi_{\min }=-29, \psi_{\max }=57.6, \Delta \psi \cong 6.2$, streamlines are shown in eight snapshots evenly distributed during the timeframe $\tau_{\omega}=2 \pi / \varpi$ ).

An increase in the Rayleigh number from $\mathrm{Ra}_{\omega}=3 \times 10^{4}$ to $10^{5}$, does not change the general qualitative behaviour. Two disturbances propagating in opposite directions can be still clearly identified (Figure 11). Some quantitative changes, however, must be taken into account. Indeed, it can be seen that the convective disturbance of buoyant nature is now strong enough to cause the emergence of clockwise oriented cells in some stages during a cycle of modulation (more precisely, when the vibration-induced acceleration is directed from the bottom wall towards the free surface, i.e. in positive y direction; frames (a-c)). Buoyancy also makes the disturbance travelling downstream the dominant mode of convection when the acceleration is reversed (acceleration directed from the surface towards the bottom wall, i.e. in the negative y direction; frames (d-h)). 


\section{$\underline{\text { E. Resonant States }}$}

In this section we briefly investigate the possible existence of "resonances", i.e. of special convective (resonant) states in which the frequency of the mechanical vibrations is equal to the "natural" frequency related to an oscillatory instability of the "base" flow (in our case the travelling HTW that occurs in the unmodulated case).

This may be regarded as a special case of situations where the frequency of forcing and the frequency of the hydrothermal wave related to Marangoni flow have the same order of magnitude (see the simulations discussed in Sect. IIID for $\varpi=10^{2}$ ).

It is shown here how special types of spatiotemporal resonances can arise for $\varpi=\Omega_{\mathrm{HTw}}$ because this condition permits selection of different interacting spatial modes. Accordingly, a wider flexibility in possible oscillatory behaviours (which can form) is allowed.
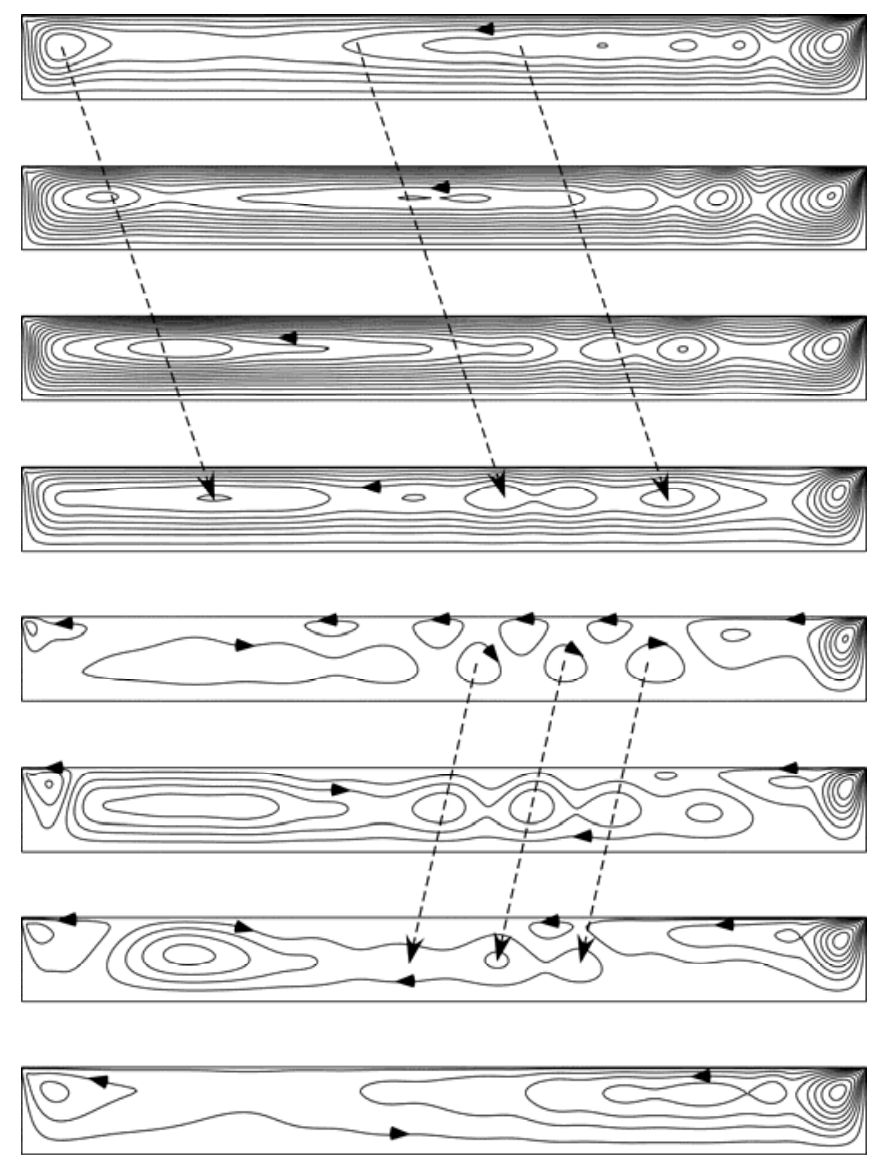

Figure 12: Mixed Marangoni-vibrational flow (Ma=3x10 ${ }^{4}, \varpi=\Omega_{\mathrm{HTw}}, \mathrm{Ra}_{\omega}=3 \times 10^{4}, \mathrm{~B}_{\omega}=\mathrm{Ra}_{\omega} / \mathrm{Ma}=1$, $\theta=0$, vibrations parallel to liquid/gas interface, $\psi_{\min }=-27.4, \psi_{\max }=73, \Delta \psi \cong 5.9$, streamlines are shown in eight snapshots evenly distributed during the timeframe $\left.\tau_{\mathrm{HTW}}\right)$.

Along these lines, Figure 12 indicates that when the case of horizontal vibrations is considered, although the dynamics resemble those already discussed for $\varpi=10^{2}$ (with the periodic emergence of a region of reversed flow and the interface), the pattern displays a richer variety of oscillatory 
modes. Indeed, two main stages of oscillatory behaviour can be observed in a period $2 \pi / \varpi$, with rolls propagating in the upstream direction at the beginning (frames (a-d)) as expected for pure Marangoni flow, and then moving in the opposite direction when the main circulation system driven by the joint action of Marangoni effects and buoyancy is taken over by the elongated clockwise oriented cell responsible for the reversed flow at the interface.

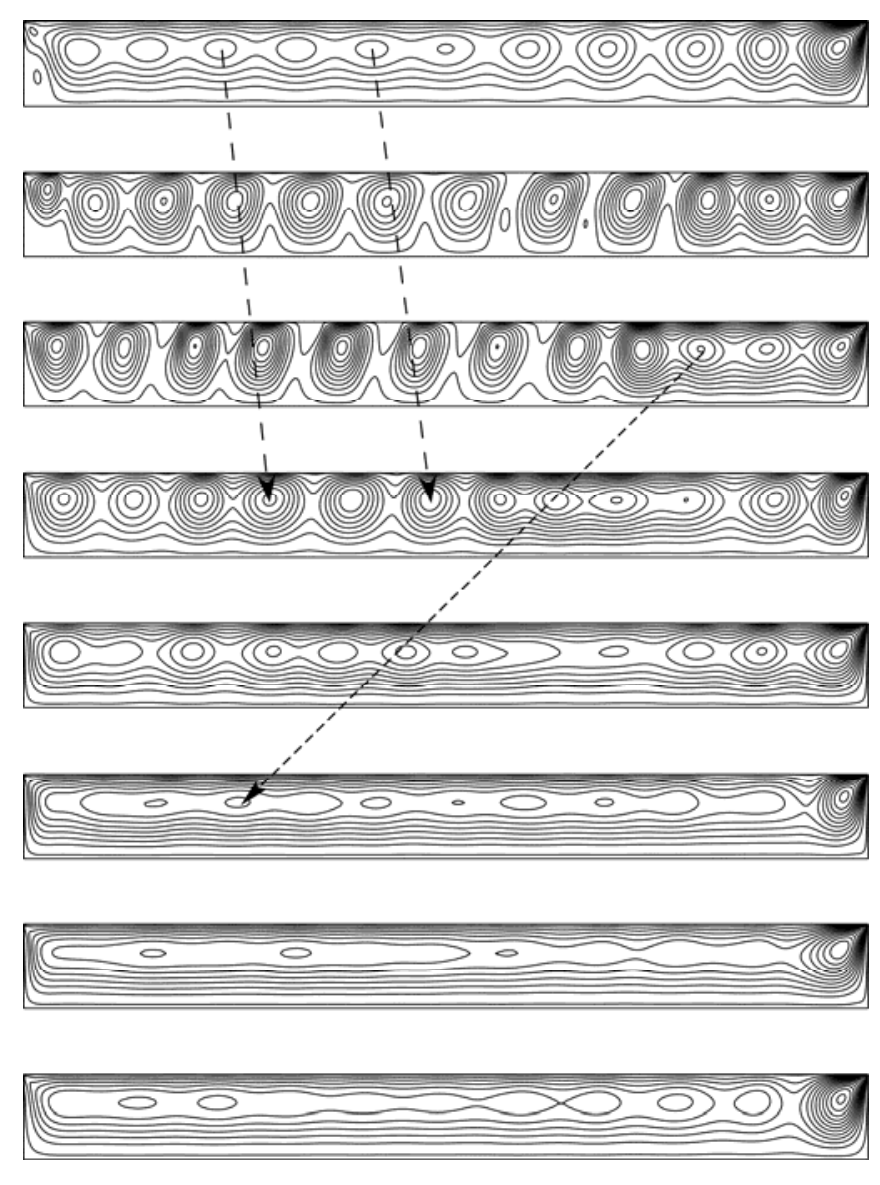

Figure 13: Mixed Marangoni-vibrational flow (Ma=3x10 ${ }^{4}, \varpi=\Omega_{\mathrm{HTW}}, \mathrm{Ra}_{\omega}=3 \times 10^{4}, \mathrm{~B}_{\omega}=\mathrm{Ra}_{\omega} / \mathrm{Ma}=1$, $\theta=\pi / 2$, vibrations perpendicular to liquid/gas interface, $\psi_{\min }=-7.7, \psi_{\max }=49.5, \Delta \psi \cong 4.1$, streamlines are shown in eight snapshots evenly distributed during the timeframe $\tau_{\mathrm{HTW}}$ ).

Again, two counteracting effects can be observed in the case of vibrations perpendicular to the interface (Figure 13). Rolls propagate in the upstream direction as in the vibration-less case, but their motion is slowed down by a disturbance periodically emerging at the hot size and propagating to the other (cold) side over a period. Such a disturbance causes a weakening of the rolls it meets, and, at the same time, it promotes the formation of a single elongated cell (via the coalescence of weakened component rolls).

Correspondingly, isotherms display a very interesting alternance of almost horizontal isotherms (vertical stratification induced by the Marangoni effect) and plumes (of Rayleigh-Bénard origin) originating from the bottom wall due to buoyancy. 


\section{Discussions}

Having finished a description of the patterning behaviour and some related quantitative details (as a function of vibration direction, frequency and Rayleigh number) we now turn to interpreting the results described in the previous sections under an alternate point of view that may provide a more rigorous characterization and classification of all solutions. In particular, we will show how the problem becomes at once more manageable and more intuitive if it is cast in the form of maps in the phase space.

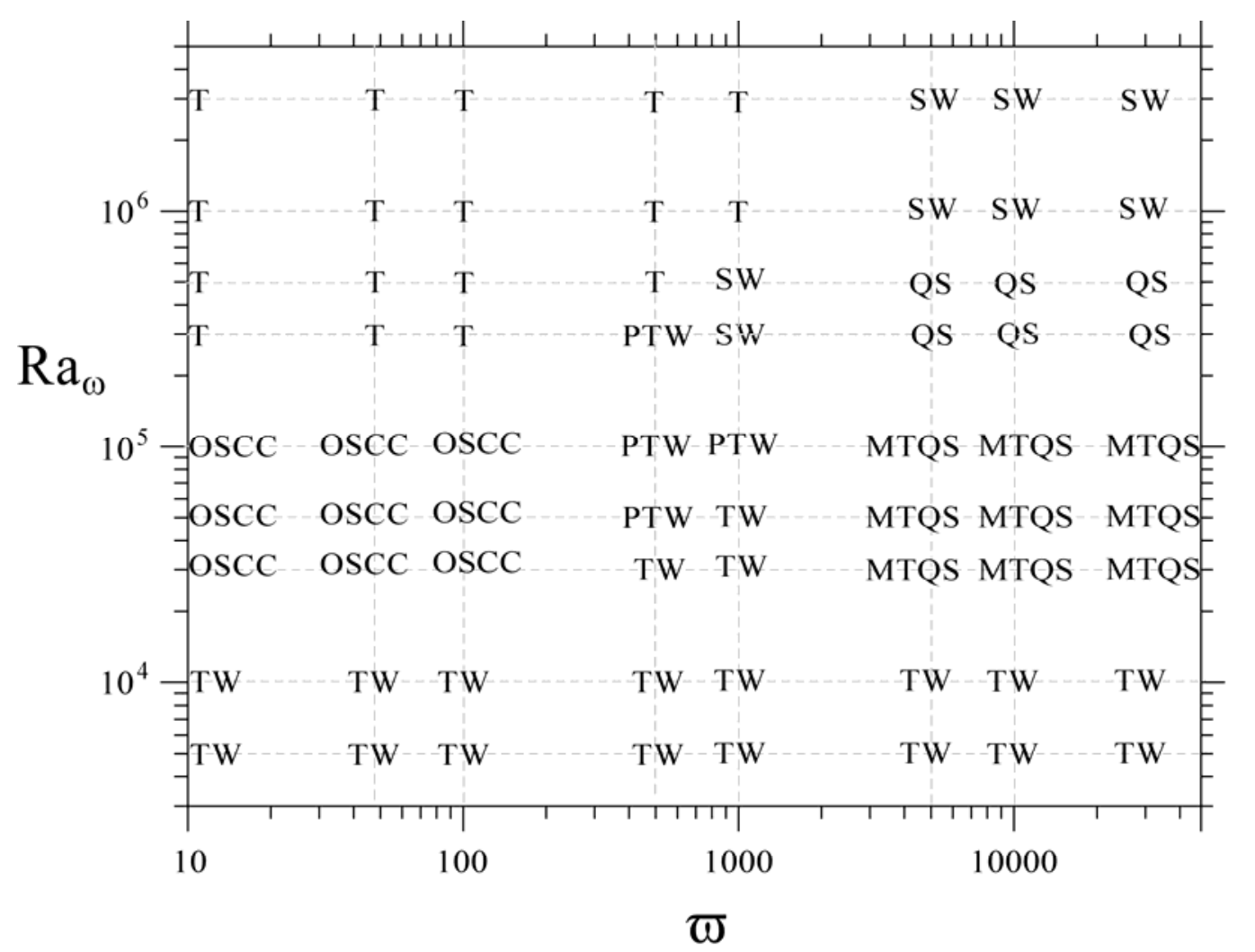

Figure 14: Map of spatiotemporal states and waveforms as a function of acceleration amplitude and frequency in the case of vibrations parallel to the layer $\left(\theta=0^{\circ}\right)$. Legend: Oscillatory State with Counter-rotating Cell (OSCC); Upstream Travelling Wave (TW); Pulso-Travelling (Upstream) Wave (PTW); Standing Wave (SW); Quasi-stationary State (QS); Mixed Travelling Quasistationary State (MTQS); Turbulence (T).

A summary in the case of vibrations directed along a direction parallel to the liquid layer is shown in Fig. 14. In this map, a diagram with $\varpi$ as abscissa and $\mathrm{Ra}_{\omega}$ as ordinate, three distinct regions can be clearly discerned. The first one is the region of "standard" HTW emergence, that is the area of the space of parameters $\left(\mathrm{Ra}_{\omega}, \varpi\right)$ where the hydrothermal wave produced by Marangoni flow seems to be unaffected (at least from a qualitative standpoint) by the presence of vibrations (regardless of their acceleration amplitude and frequency). From a purely technical standpoint, this region would correspond to "safe" conditions for the execution of an ideal space experiment aimed at studying the formation mechanism of hydrothermal waves induced by Marangoni flow. 
The opposite situation, i.e. the region in which a flow having clearly recognizable characteristics can no longer be identified (turbulent conditions), is also present in Figure 14 (this should be regarded as the worst case as opposed to the ideal situation discussed before).

A region also exists, which separates the two limit situations above. As the reader may easily realize, this is region where the interesting and exotic dynamics described in the earlier sections occur.

Here, in the direction of identifying in a unique and consistent way the spatio-temporal behaviour of the emerging oscillatory solution when initial disturbances saturate their amplitude, we classify definitely the solutions as: travelling (TR), standing (SR) rolls solutions (equivalent to travelling (TW) and standing (SW) waves, respectively), quasi-stationary (QS) convection and "mixed" states, namely patterns which display at the same time features pertaining to more than one of these fundamental modes of convection. No definition is perfect, and it is hard to disentangle a definition from a property, but the following categorization captures the essential aspects of the observed phenomena.

By the first category we intend solutions where a clear direction of propagation of the fluiddynamic disturbance can be identified. The second type of flow consists of rolls "pulsating” at fixed positions along the horizontal extension of the layer (undergoing a remarkable periodic growth and decay in time and eventually a change in the sense of rotation).

This pattern shows an appearance that is totally different from the travelling wave. In physics, a standing wave, is a wave (a disturbance oscillating in time) that remains in a constant position. In general, this phenomenon emerges as a result of the interference (superposition) of two waves having the same amplitude and same angular frequency $\omega$, but travelling in opposite directions. This typically results in a field with no net disturbance transport on average along the propagation direction of the two component waves (the $\mathrm{x}$ axis in the present case for which we consider a liquid layer). In terms of patterning behaviour, the final effect is a series of nodes (disturbance zero amplitude) and anti-nodes (disturbance maximum amplitude) at fixed locations along x.

The essentially stationary nature of the resulting pattern distinguishes the third case (QS convection) from the other phenomena. It is termed in this way because of the gently "fluctuating" nature of rolls, which change neither their position along $\mathrm{x}$, nor their sense of rotation.

The last category is given by patterns with a more or less regular spatio-temporal behaviour that can be seen as a combination of portions of patterns pertaining to different fundamental classes or as states displaying the fundamental properties of two different variants at the same time. A typical example along these lines is the "pulso-travelling mode" (PTW), i.e. rolls that undergo a change in strength while they migrate along the $\mathrm{x}$-direction. 


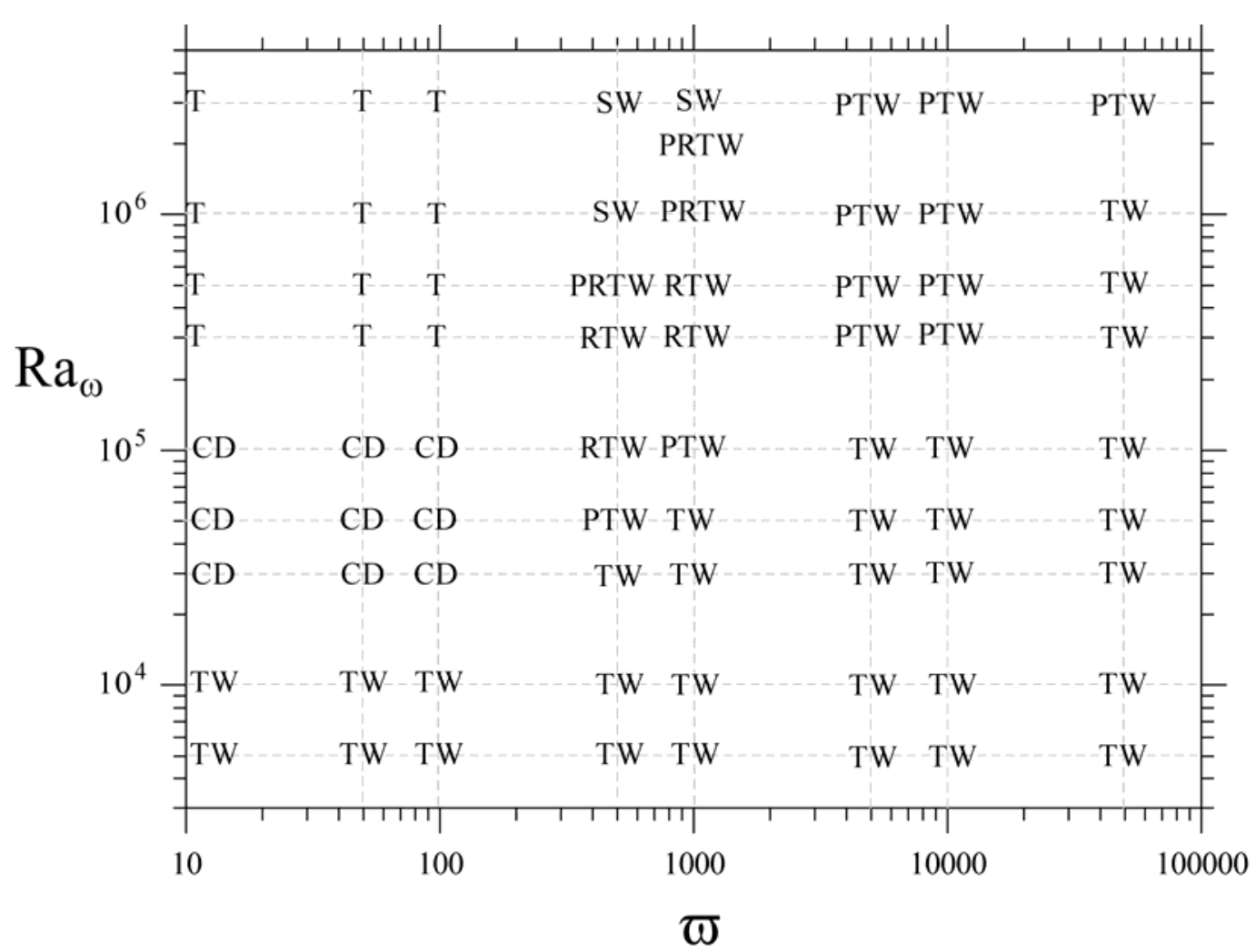

Figure 15: Map of spatiotemporal states and waveforms as a function of acceleration amplitude and frequency in the case of vibrations perpendicular to the layer $\left(\theta=90^{\circ}\right)$. Legend: Counter-propagating Disturbances (CD); Upstream Travelling Wave (TW); Reversed Travelling Wave [this wave travels in the downstream direction] (RTW); Pulso-Travelling Wave (PTW); Pulso-Travelling Reversed Wave (PRTW); Standing Wave (SW); Turbulence (T).

A direct comparison of Fig. 15, summarizing the results for the case in which the angle formed by g-jitters and free liquid/gas interface is $\theta=90^{\circ}$, with Fig. 14 leads to the more or less immediate realization that vibrations perpendicular to the liquid layer are, in general, less "dangerous" than those acting along the $\mathrm{x}$ axis. The region of classical HTW occurrence is much more extended with respect to the case $\theta=0^{\circ}$. Also, a notable shrinkage of the region of turbulent behaviour can be observed.

Interestingly, in such a case some additional varieties (in terms of characteristics of the emerging pattern) occur which are not present in the $\theta=0^{\circ}$ case; we refer, in particular, to Figs. 6-7 and 10-11, which lead to the definition of two new categories of patterns as further described below.

The first class (Fig. 6-7) is hereafter referred to as "reversed travelling wave" (RTW) owing to the sense of propagation of the disturbances, which now travel continuously in the downstream direction.

The second new variant is the "mixed-traveling mode" (denoted by CD in Fig. 15), i.e. the oscillatory state resulting from the coexistence of two distinct disturbances traveling in opposite directions with different amplitudes and angular velocities (Figs. 10-11). 
This second type of solution can be met moving along the vertical traverse at $\varpi=100$. The standard HTW existing for $\mathrm{Ra}_{\omega}<10^{4}$ is replaced by the mixed-traveling mode with counterpropagating disturbances for $10^{4}<\mathrm{Ra}_{\omega}<10^{5}$ before a transition to turbulence occurs at higher values of $\mathrm{Ra}_{\omega}$.

These specific dynamics may be seen as a consequence of the increased ability of inertial disturbances to interfere with the delicate mechanisms supporting the HTW when they are perpendicular to the layer and their angular frequency is comparable to the angular frequency of the hydrothermal wave, i.e. when these two quantities have the same order of magnitude $O(\varpi)=O(\Omega)$.

Indeed, an increase in $\varpi$ at constant $\mathrm{Ra}_{\omega}=10^{5}$ beyond $\varpi=3 \times 10^{2}$, determines a suppression of this fascinating behaviour, with the emergence of a pulso-travelling mode at $\varpi=10^{3}$ and recovery of a classical HTW at $\varpi=10^{4}$.

Even more interesting are the dynamics encountered when moving along a vertical traverse at $\varpi=10^{3}$. For $\operatorname{Ra}_{\omega}>3 \times 10^{4}$, the fairly regular classical HTW (see, e.g., Fig. 2) is replaced by a pulsotravelling mode at $\mathrm{Ra}_{\omega}=10^{5}$; a further increase in $\mathrm{Ra}_{\omega}$, however, determines a change in the sense of propagation of the disturbance thereby producing the aforementioned "reversed travelling wave" (Figs. 6-7).

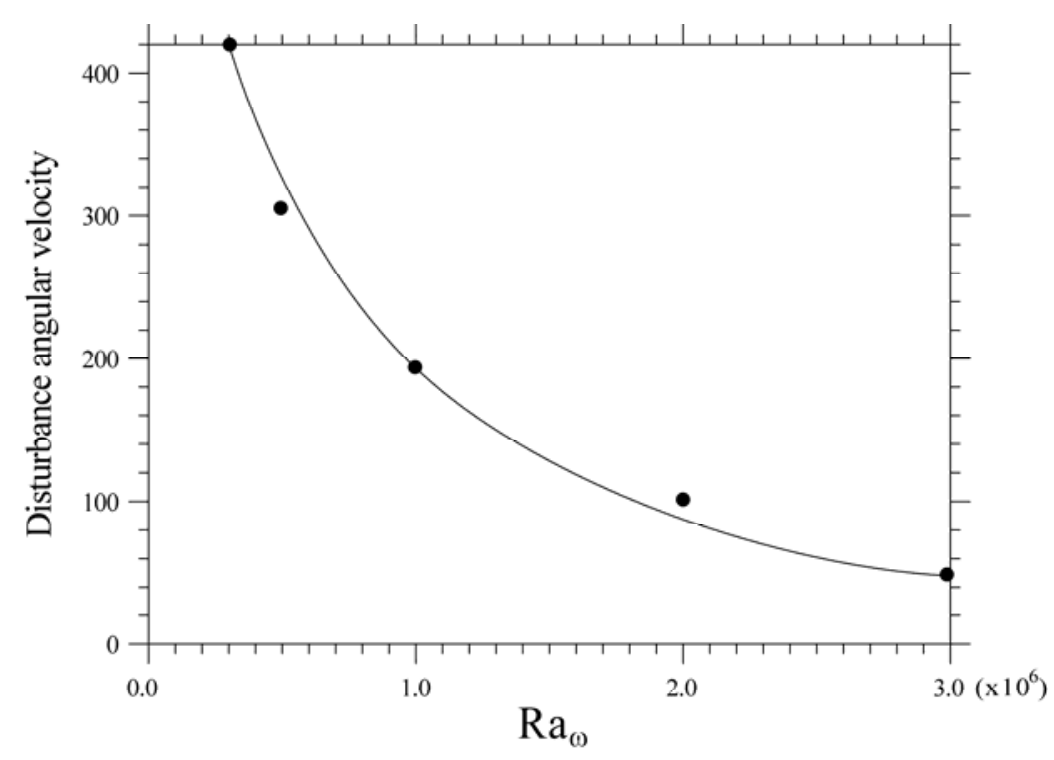

Figure 16: Reversed travelling wave - Disturbance Angular velocity (absolute value) as a function of $\mathrm{Ra}_{\omega}$ for $\varpi=10^{3}$ (the solid line is a polynomial fit with degree 2 indicating that in this regime the disturbance angular velocity decreases quadratically with the Rayleigh number).

Given the strong increase displayed by the related angular velocity (in comparison to the pure Marangoni flow) when the change in the sense of propagation occurs, it can be argued that the disturbance is essentially thermovibrational in nature. Its sense of propagation, however, must be still ascribed to the presence of Marangoni flow.

The general principle established by the linear stability analysis by Smith and Davis [36] implies that in the case of a basic-state flow-induced temperature distribution along y corresponding to a positive vertical temperature gradient, disturbances should always travel in a direction with a 
component in the direction opposite to that of the surface flow. Albeit, the wave travelling downstream clearly violates this principle, one must keep in mind that the base state in the cavity is characterized not only by a vertical temperature gradient but also by the tendency to transport fluid along the interface from the hot side to the cold side due to the Marangoni surface stresses. By breaking the in-plane isotropy that would characterize pure thermovibrational flow, the presence of these stresses may explain the observed sense of propagation of rolls.

Beyond $\mathrm{Ra}_{\omega}=10^{6}$, this solution displays again the typical properties of a mixed mode with rolls traveling and pulsating at the same time. A further increase in $\operatorname{Ra}_{\omega}$ strengthens the pulsating aspect of the rolls (Fig. 8) with respect to their traveling nature (their velocity of propagation tends to be reduced as $\mathrm{Ra}_{\omega}$ is increased, Fig. 16) until a pulsating-only behaviour (standing wave) is attained for $\operatorname{Ra}_{\omega} \geq 3 \times 10^{6}$.

At this stage, it is also worth considering some comparison with some "similar" results available in the literature. Notably, Zebib [27] found vertical modulation in the limit of high frequencies (i.e. the Gershuni's approximation) to stabilize the hydrothermal branch for any value of Pr. The influence of vibrations on the hydrothermal waves was reported as a function of a dimensionless parameter (W) defined as:

$W=\frac{\widetilde{R} a_{\omega}^{2} \operatorname{Pr}}{2 \widetilde{M} a \varpi^{2}}=\frac{\Lambda^{2} \varpi^{2}}{2 \widetilde{M} a \operatorname{Pr}}$

where $\tilde{M} a=\sigma_{\mathrm{T}} \gamma \mathrm{d}^{2} / \mu \alpha$ and $\tilde{R} a_{\omega}=\mathrm{b} \omega^{2} \beta_{\mathrm{T}} \gamma \mathrm{d}^{4} / \nu \alpha=\Lambda \Omega^{2} / \mathrm{Pr}$, respectively, where $\gamma$ is the rate of imposed uniform temperature increase along the $\mathrm{x}$ axis $(\Delta \mathrm{T} / \ell$ in our case).

The hydrothermal waves were observed to be stabilized (i.e. to emerge at higher values of Ma) with increasing W. Most interestingly, in theoretical agreement with the present results the waves could reverse their direction of propagation, i.e. travel in the same direction as the free surface flow at particular values of $\mathrm{W}$ depending on $\mathrm{Pr}$.

For $\operatorname{Pr}=15$, W was found to be $\cong 1.5 \times 10^{-1} \rightarrow$

$\widetilde{R} a_{\omega}^{2} \cong 10^{-2} \tilde{M} a \varpi^{2}$

which, replacing $\tilde{M} a$ and $\tilde{R} a_{\omega}$ with the equivalent expressions in terms of our Ra and Ma, reads:

$R a_{\omega}^{2} \cong 10^{-2} \tilde{M} a \varpi^{2} A=2 \times 10^{-1} M a \varpi^{2}$

For $\mathrm{Ma}=3 \times 10^{4}$ and $\varpi=10^{3}$, this relationship gives finally a transition Rayleigh number $\mathrm{Ra}_{\omega} \cong 10^{5}$, which is in good agreement with the present findings (wave travelling in the downstream direction emerging only for $\mathrm{Ra}_{\omega}>\mathrm{O}\left(10^{5}\right)$. 


\section{Conclusions}

We have focused on the patterning behaviour and oscillatory response of a plane liquid layer supporting Marangoni flow to the application of inertial disturbances of given intensity and frequency. In particular, attention has been concentrated on a traveling wave (HTW), which of Marangoni convection represents a well-known solution.

The hydrothermal wave appears as a train of rolls moving continuously from the cold side towards the hot one. This relatively simple description, however, is no longer applicable as soon as complications, such as external periodic perturbations of inertial nature are added to the considered problem.

These perturbations can prevent HTW formation at all. In general, however, there exist, between this limiting classical solution and turbulent behaviours in space (and/or time), numerous intermediate situations. In particular, on the basis of the present numerical results it is possible to divide the regimes of modulated supercritical Marangoni convection into three main regions: (i) apparently unaffected HTWs, (ii) hybrid thermocapillary-thermovibrational waves, (iii) turbulent convection. The second region, in turn, can be further partitioned into a zoo of possible modes of convection.

The numerical simulations have shown how, in general, the behaviour at a large scale of the system and the resulting properties of the flow arise from detailed structures on different scales. In such a context, some effort has been directed to separate expressly on such scales the features which characterize the pattern and create its recognizable identification. Accordingly, solutions have been classified in detail as: travelling, standing (pulsating), quasi-stationary and "mixed" modes. This last category, in turn, has been split into different variants; among them: a "pulso-travelling mode”, i.e. rolls that undergo a change in strength while they migrate along the x-direction and a "mixedtraveling mode", i.e. the oscillatory state resulting from the coexistence of two distinct disturbances traveling in opposite directions with different amplitudes and angular velocities.

We have found that both large and small rolls, appearing periodically, can affect the dynamics at small values of the vibrations nondimensional frequency. A more precise assessment of the impact of vibrations frequency $(\varpi)$ and intensity $\left(\operatorname{Ra}_{\omega}\right)$, however, has revealed that for a fixed value of $\operatorname{Ra}_{\omega}$ a first regime exists where large-scale disturbances are the dominant mode of convection. As this frequency attains progressively larger values, the large-scale disturbance is taken over by disturbances on a smaller scale. An additional increase in $\varpi$ tends to suppress also these dynamics leading to a full recovery of the classical HTW. By contrast, increases in $\mathrm{Ra}_{\omega}$ at a constant value of $\varpi$ cause, in general, a transition to turbulence at relatively small values of $\varpi$ or a tendency to replace travelling waves with pulsating or stationary modes at larger $\varpi\left(\geq 10^{3}\right)$.

We have proved that it is somehow possible to take advantage of modulation of Marangoni convection induced by physical vibrations of the overall system: 1) to induce a mitigation of the resulting convective disturbances; 2) to control convection patterning (i.e. induce spatiotemporal 
convective patterns with desired features); and 3) to induce changes in the system hierarchy of bifurcations (modify threshold parameters and features of the supercritical states).

\section{References}

[1] M. Lappa, Fluids, Materials and Microgravity: Numerical Techniques and Insights into the Physics, (Elsevier Science, Oxford, 2004), 538pp. - ISBN 00-804-4508-X.

[2] R. Monti and R. Savino, Microgravity experiment acceleration tolerability on space orbiting laboratories, J. Spacecraft Rockets 33(5), 707-716, (1996).

[3] I.B. Simonenko and S.M. Zen'kovskaja, On the effect of high-frequency vibrations on the origin of convection, Izv. Akad. Nauk SSSR. Ser. Meh. Zhidk. Gaza, 5: 51-55, (1966).

[4] A. Zebib, G.M. Homsy, and E. Meiburg, High Marangoni number convection in a square cavity, Phys. Fluids, 28: 3467-3476, (1985).

[5] E. Bucchignani, Numerical characterization of hydrothermal waves in a laterally heated shallow layer, Phys Fluids, 16(11): 3839-3849, (2004).

[6] F. Sato, I. Ueno, H. Kawamura, K. Nishino, S. Matsumoto, M. Ohnishi, M. Sakurai, Hydrothermal wave instability in a high-aspect-ratio liquid bridge of $\operatorname{Pr}>200$, Microgravity Sci. Technol.,. 25: 43-58, (2013).

[7] D. Schwabe, Convective instabilities in complex systems with partly free surface, Journal of Physics:Conference Series 64, 012001, (2007).

[8] T.P. Lyubimova, A . Lizee, G.Z. Gershuni, D.V. Lyubimov, G. Chen, M. Wadih, B. Roux, High frequency vibrations influence on heat transfer, Microgravity Q., 4 (4): 259-268, (1994).

[9] K. K. Gabdrakhmanov and V. G. Kozlov, Experimental investigation of thermo-oscillatory convection, Fluid Dyn., 37 (3), 369-376, (2002).

[10] R. Savino and M. Lappa, Assessment of the thermovibrational theory: application to g-jitter on the Space-station, J. Spacecraft Rockets, 40(2), 201-210, (2003).

[11] A. Mialdun, I. I. Ryzhkov, D. E. Melnikov and V. Shevtsova, Experimental evidence of thermalvibrational convection in a nonuniformly heated fluid in a reduced gravity environment, Phys. Rev. Lett .,101, 084501, (2008).

[12] V. Shevtsova, Y. Gaponenko, V. Sechenyh, D. Melnikov, T. Lyubimova and A. Mialdun, Dynamics of a binary mixture subjected to a temperature gradient and oscillatory forcing, $J$. Fluid Mech., 767, 290- 322, (2015).

[13] P.M. Parmentier, V.C. Regnier and G. Lebon, Buoyant-thermocapillary instabilities in medium-Prandtl number fluid layers subject to a horizontal temperature gradient, Int. J. Heat Mass Transfer, 36: 2417-2427, (1993).

[14] J. Burguete, N. Mukolobwiez, N. Daviaud, N. Garnier, A. Chiffaudel, Buoyantthermocapillary instabilities in extended liquid layers subjected to a horizontal temperature gradient, Phys. Fluids, 13 (10), 2773-2787, (2001). 
[15] A. A. Nepomnyashchy and I. B. Simanovskii, Combined action of different mechanisms of instability in multilayer systems, Phys. Rev. E, 59, 6672, (1999).

[16] A. A. Nepomnyashchy and I. B. Simanovskii, Convective flows in a two-layer system with a temperature gradient along the interface, Phys. Fluids 18, 032105, (2006).

[17] V.M. Shevtsova, A.A. Nepomnyashchy and J.C. Legros, Thermocapillary-buoyancy convection in a shallow cavity heated from the side, Phys. Rev. E, 67, 066308 (14 pages), (2003)

[18] A. Nepomnyashchy, I. Simanovskii and L. Braverman, Convective oscillations in multilayer systems under the combined action of buoyancy and thermocapillary effect, Phys. Fluids, 17, 022103, (2005).

[19] R.V. Birikh, V.A. Briskman, V.I. Chernatynski, B. Roux, Control of thermocapillary convection in a liquid bridge by high frequency vibrations, Microgravity Q., 3, 23-28, (1993).

[20] R.V. Birikh, V.A. Briskman, A.L. Zuev, V.I. Chernatynskii and V.I. Yakushin, Interaction of the thermovibrational and thermocapillary convection mechanisms, Fluid Dynamics, 29(5), 681-692, (1994). Translated from Izvestiya Rossiiskoi Akademii Nauk, Mekhanika Zhidkosti i Gaza, No. 5, pp. 107-121, September-October, 1994.

[21] P. Grassia and G. M. Homsy, Thermocapillary and buoyant flows with low frequency jitter. I. Jitter confined to the plane, Phys. Fluids, 10, 1273-1290, (1998).

[22] P. Grassia and G. M. Homsy, Thermocapillary and buoyant flows with low frequency jitter. II. Spanwise jitter, Phys. Fluids, 10, 1291-1314, (1998).

[23] V. A. Suresh, C.I. Christov and G.M. Homsy, Resonant thermocapillary and buoyant flows with finite frequency gravity modulation, Phys. Fluids, 11, 2565-2576, (1999).

[24] E.T. Tudose and M. Kawaji, An Experimental Study of Thermocapillary Convection in a Thin Horizontal Liquid Layer in a Rectangular Cavity under Normal Gravity with and without Controlled Vibration, Paper IAF-99-J.4.08, presented at the 50th International Astronautical Congress, October 4-8, 1999, Amsterdam, The Netherlands.

[25] V. Suresh and G.M. Homsy, Stability of return thermocapillary flows under gravity modulation, Phys. Fluids, 13, 3155-3167, (2001).

[26] T.P. Lyubimova, R.V. Scuridin, A. Croell, P. Dold, Influence of high frequency vibrations on fluid flow and heat transfer in a floating zone, Cryst. Res. Technol., 38 (7-8), 635-653, (2003).

[27] A. Zebib, Vibrational thermocapillary instabilities, J. Fluid Mech., 540: 353-371, (2005).

[28] O. Gamache and M. Kawaji, Experimental Investigation of Marangoni Convection and Vibration-Induced Crystal Motion during Protein Crystal Growth, Microgravity Science and Technology, XVI-I, 342-347, (2005).

[29] S.M. Zen'kovskaya, V.A. Novosyadlyia and A.L. Shleikel', The effect of vertical vibration on the onset of thermocapillary convection in a horizontal liquid layer, Journal of Applied Mathematics and Mechanics, 71(2), 247-257, (2007).

[30] M. Lappa, Thermal Convection: Patterns, Evolution and Stability (John Wiley \& Sons, Chichester, England, 2009). 
[31] M. Lappa, Control of convection patterning and intensity in shallow cavities by harmonic vibrations, Microgravity Science \& Technology, To appear - DOI: 10.1007s12217-015-94674, (2015)

[32] G. Z. Gershuni and E. M. Zhukhovitskii, Free thermal convection in a vibrational field under conditions of weightlessness, Sov. Phys. Dokl., 24 (11), 894-896, (1979).

[33] G. Z. Gershuni and E. M. Zhukhovitskii, Vibrational thermal convection in zero gravity, Fluid. Mech. Sov. Res., 15 (1), 63-84, (1986).

[34] M. Lappa, Three-dimensional numerical simulation of Marangoni flow instabilities in floating zones laterally heated by an equatorial ring, Phys. Fluids, 15 (3), 776-789, (2003).

[35] M. Lappa, Combined effect of volume and gravity on the three-dimensional flow instability in non-cylindrical floating zones heated by an equatorial ring, Phys. Fluids, 16 (2), 331-343, (2004).

[36] M.K. Smith and S.H. Davis, Instabilities of dynamic thermocapillary liquid layers. Part 1: convective instabilities, J. Fluid Mech, 132, 119-144, (1983).

[37] L. Peltier and S. Biringen, Time-dependent thermocapillary convection in a rectangular cavity: numerical results for a moderate Prandtl number fluid, J. Fluid Mech., 257, 339-357, (1993).

[38] J. Xu and A. Zebib, Oscillatory two- and three-dimensional thermocapillary convection, $J$. Fluid Mech., 364, 187-209, (1998).

[39] Z.M. Tang and W.R. Hu, Hydrothermal Wave in a Shallow Liquid Layer, Microgravity Sci. Tech., 16(1), 253-258, (2005). 